\title{
New perspectives on covariant quantum error correction
}

\author{
Sisi Zhou ${ }^{1,2}$, Zi-Wen Liu ${ }^{3}$, and Liang Jiang ${ }^{2}$ \\ ${ }^{1}$ Department of Physics, Yale University, New Haven, Connecticut 06511, USA \\ ${ }^{2}$ Pritzker School of Molecular Engineering, The University of Chicago, Illinois 60637, USA \\ ${ }^{3}$ Perimeter Institute for Theoretical Physics, Waterloo, Ontario N2L 2Y5, Canada
}

Covariant codes are quantum codes such that a symmetry transformation on the logical system could be realized by a symmetry transformation on the physical system, usually with limited capability of performing quantum error correction (an important case being the Eastin-Knill theorem). The need for understanding the limits of covariant quantum error correction arises in various realms of physics including fault-tolerant quantum computation, condensed matter physics and quantum gravity. Here, we explore covariant quantum error correction with respect to continuous symmetries from the perspectives of quantum metrology and quantum resource theory, establishing solid connections between these formerly disparate fields. We prove new and powerful lower bounds on the infidelity of covariant quantum error correction, which not only extend the scope of previous no-go results but also provide a substantial improvement over existing bounds. Explicit lower bounds are derived for both erasure and depolarizing noises. We also present a type of covariant codes which nearly saturates these lower bounds.

\section{Introduction}

Quantum error correction (QEC) is a standard approach to protecting quantum systems against noises, which for example allows the possibility of practical quantum computing and has been a central research topic in quantum information [1-3]. The key idea of QEC is to encode the logical state into a small code subspace in a large physical system and correct noises using the redundancy in the entire Hilbert space. As a result, the structure of noise must also place restrictions on QEC codes. This feature was beautifully captured by the Eastin-Knill theorem [4] (see also [5-8]), which states that any non-trivial local-error-correcting quantum code does not admit transversal implementations of a universal set of logical gates, ruling out the possibility of realizing faulttolerant quantum computation using only transversal gates.

In particular, any finite-dimensional local-error-correcting quantum code only admits a finite number of transversal logical operations, which forbids the existence of codes covariant with continuous symmetries (discrete symmetries are allowed though [9, 10]). More generally, quantum codes under symmetry constraints, namely covariant codes, are of great practical and theoretical interest. In general, a quantum code is covariant with respect to a logical Hamiltonian $H_{L}$ and a physical Hamiltonian $H_{S}$ if any symmetry transformation $e^{-i H_{L} \theta}$ is encoded into a symmetry transformation $e^{-i H_{S} \theta}$ in the physical system. Besides important implications to fault-tolerant quantum computation, covariant QEC is also closely connected to many other topics in quantum information and physics, such as quantum reference frames and quantum clocks $[9,11,12]$, symmetries in the AdS/CFT correspondence [10, 12-18] and approximate QEC in condensed matter physics [19]. Although covariant codes cannot be perfectly local-error-correcting, they can still approximately

Sisi Zhou: sisi.zhou26@gmail.com

Zi-Wen Liu: zliu1@perimeterinstitute.ca

Liang Jiang: liang.jiang@uchicago.edu 
correct errors with the infidelity depending on the number of subsystems, the dimension of each subsystem, etc. The quantifications of such infidelity in covariant QEC were explored recently, leading to an approximate, or robust, version of the Eastin-Knill theorem [10, 12], using complementary channel techniques [20-22]. Note that these existing results only apply to erasure errors and random phase errors at unknown locations.

In this paper, we investigate covariant QEC from the perspectives of quantum metrology and quantum resource theory, which not only establishes conceptual and technical links between these seemingly separate fields, but also leads to a series of improved understandings and bounds on the performance of covariant QEC. Quantum metrology studies the ultimate limit on parameter estimation in quantum systems [23-27]. Covariant QEC is naturally a quantum metrological protocol - estimating the angle of any rotation of the physical system is equivalent to estimating that of the logical system with protection against noise. There is a no-go theorem in quantum metrology stating that perfectly error-correcting codes admitting a non-trivial logical Hamiltonian do not exist if the physical Hamiltonian falls into the Kraus span of the noise channel, which is known as the HKS condition [28-34]. It is also a sufficient condition of the non-existence of perfectly covariant QEC codes. When the HKS condition is satisfied, we establish a connection between the quantum Fisher information (QFI) of quantum channels [30, 34-38] and the performance (or infidelity) of covariant QEC, which gives rise to the desired lower bound. We could also understand covariant QEC in terms of the resource theory of asymmetry [39-41] with respect to translations generated by Hamiltonians, where the covariant QEC procedures may naturally be represented by free operations. In quantum resource theory, we also have no-go theorems which dictate that pure resource states cannot be perfectly distilled from generic mixed states [42-44], thereby ruling out the possibility of perfect covariant QEC. By further analyzing suitable resource monotones, in particular a type of QFI [44], we derive a lower bound on the infidelity of covariant QEC, which behaves similarly to the metrological bounds.

Our approaches and results on covariant QEC are innovative and also advantageous compared to previous ones in many ways. The bounds generalize the no-go theorems for covariant QEC from local Hamiltonians with erasure errors to generic Hamiltonian and noise structures. In the special case of erasure noise, our lower bounds improve the previous results in the small infidelity limit [10]. Furthermore, we demonstrate that there is a type of covariant codes called thermodynamic codes $[10,19]$ that saturates the lower bound for erasure noise and matches the scaling of the lower bound for depolarizing noise, while previous bounds only apply to the erasure noise setting and were not known to be saturable [10].

\section{Preliminaries: Covariant codes}

A quantum code is a subspace of a physical system $S$, usually defined by the image of an (usually isometric) encoding channel $\mathcal{E}_{S \leftarrow L}$ from a logical system $L$. We call a code $\mathcal{E}_{S \leftarrow L}$ covariant if there exists a logical Hamiltonian $H_{L}$ and a physical Hamiltonian $H_{S}$ such that

$$
\mathcal{E}_{S \leftarrow L} \circ \mathcal{U}_{L, \theta}=\mathcal{U}_{S, \theta} \circ \mathcal{E}_{S \leftarrow L}, \forall \theta \in \mathbb{R},
$$

where $\mathcal{U}_{L, \theta}\left(\rho_{L}\right)=e^{-i H_{L} \theta} \rho_{L} e^{i H_{L} \theta}$ and $\mathcal{U}_{S, \theta}\left(\rho_{S}\right)=e^{-i H_{S} \theta} \rho_{S} e^{i H_{S} \theta}$ are the symmetry transformations on the logical and physical systems, respectively. We assume that the dimensions of the physical and logical systems $d_{S}$ and $d_{L}$ are both finite and $H_{L}$ is non-trivial $\left(H_{L} \not \subset \mathbb{1}\right)$. For simplicity, we also assume all Hamiltonians in this paper are traceless unless stated otherwise, and we use $\Delta H_{L}$ and $\Delta H_{S}$ to denote the difference between the maximum and minimum eigenvalues of the operators.

We say a quantum code is error-correcting under a noise channel $\mathcal{N}_{S}$, if $\mathcal{N}_{S}$ is invertible inside the code subspace, i.e., if there exists a CPTP map $\mathcal{R}_{L \leftarrow S}$ such that

$$
\mathcal{R}_{L \leftarrow S} \circ \mathcal{N}_{S} \circ \mathcal{E}_{S \leftarrow L}=\mathbb{1}_{L} .
$$

We assume that the output space of the noise channel $\mathcal{N}_{S}$ is still $S$ for simplicity, although our results also apply to situations where the output system is different. The error-correcting property of a quantum code is often incompatible with its covariance with respect to continuous symmetries. One representative example is the non-existence of error-correcting codes which can simultaneously 
correct non-trivial local errors and be covariant with respect to a local $H_{S}[4,9]$. However, one may still consider approximate QEC with covariant codes $[10,12,19]$. Then a question that naturally arises is how accurate covariant codes can be against certain noises. To characterize the infidelity of an approximate QEC code, we use the worst-case entanglement fidelity $f\left(\Phi_{1}, \Phi_{2}\right)$ and the Choi entanglement fidelity [45, 46] defined by

$$
f\left(\Phi_{1}, \Phi_{2}\right)=\min _{\rho} f\left(\left(\Phi_{1} \otimes \mathbb{1}_{R}\right)(\rho),\left(\Phi_{2} \otimes \mathbb{1}_{R}\right)(\rho)\right)
$$

and

$$
f_{\text {Choi }}\left(\Phi_{1}, \Phi_{2}\right)=f\left(\left(\Phi_{1} \otimes \mathbb{1}_{R}\right)(|\gamma\rangle\langle\gamma|),\left(\Phi_{2} \otimes \mathbb{1}_{R}\right)(|\gamma\rangle\langle\gamma|)\right)
$$

for two quantum channels $\Phi_{1}$ and $\Phi_{2}$, where the fidelity between two states $\rho$ and $\sigma$ is given by $f(\rho, \sigma)=\operatorname{Tr}\left(\sqrt{\rho^{1 / 2} \sigma \rho^{1 / 2}}\right)[1]$, and $R$ is a reference system identical to the system $\Phi_{1,2}$ acts on (which we assume to be $L$ ) and the maximally entangled state $|\gamma\rangle=\frac{1}{\sqrt{d_{L}}} \sum_{i}|i\rangle_{L}|i\rangle_{R}$. After optimizing over recovery channels $\mathcal{R}_{L \leftarrow S}$, we may define the infidelity and the Choi infidelity of a code $\mathcal{E}_{S \leftarrow L}$ respectively by ${ }^{1}$

$$
\varepsilon\left(\mathcal{N}_{S}, \mathcal{E}_{S \leftarrow L}\right)=1-\max _{\mathcal{R}_{L \leftarrow S}} f^{2}\left(\mathcal{R}_{L \leftarrow S} \circ \mathcal{N}_{S} \circ \mathcal{E}_{S \leftarrow L}, \mathbb{1}_{L}\right),
$$

and

$$
\varepsilon_{\text {Choi }}\left(\mathcal{N}_{S}, \mathcal{E}_{S \leftarrow L}\right)=1-\max _{\mathcal{R}_{L \leftarrow S}} f_{\text {Choi }}^{2}\left(\mathcal{R}_{L \leftarrow S} \circ \mathcal{N}_{S} \circ \mathcal{E}_{S \leftarrow L}, \mathbb{1}_{L}\right) .
$$

Note that the Choi infidelity reflects the "average-case" behavior in the sense that it is directly related to $\varepsilon_{\text {avg }}=1-\max _{\mathcal{R}_{L \leftarrow S}} \int d \psi\left\langle\psi\left|\mathcal{R}_{L \leftarrow S} \circ \mathcal{N}_{S} \circ \mathcal{E}_{S \leftarrow L}(|\psi\rangle\langle\psi|)\right| \psi\right\rangle$, where the integral is over the Haar measure, by $\varepsilon_{\text {Choi }}=\frac{d_{L}+1}{d_{L}} \varepsilon_{\text {avg }}$. We will sometimes simply use $\varepsilon$ and $\varepsilon_{\text {Choi }}$ to denote $\varepsilon\left(\mathcal{N}_{S}, \mathcal{E}_{S \leftarrow L}\right)$ and $\varepsilon_{\text {Choi }}\left(\mathcal{N}_{S}, \mathcal{E}_{S \leftarrow L}\right)$ when the system under consideration is unambiguous. Clearly, $\varepsilon \geq \varepsilon_{\text {Choi }}$. We will use $\mathcal{R}_{L \leftarrow S}^{\text {opt }}$ to represent the optimal recovery channel achieving $\varepsilon$ and $\mathcal{I}_{L}$ to denote the effective noise channel $\mathcal{R}_{L \leftarrow S} \circ \mathcal{N}_{S} \circ \mathcal{E}_{S \leftarrow L}$ in the logical system.

\section{Metrological bound}

Recently, QEC emerges as a useful tool to enhance the sensitivity of an unknown parameter in quantum metrology [32-34, 48-57]. A good approximately error-correcting covariant code naturally provides a good quantum sensor to estimate an unknown parameter $\theta$ in the symmetry transformation $e^{-i H_{S} \theta}$. Consider a quantum signal $e^{-i H_{S} \theta}$ in the physical system, for example, the magnetic field in a spin system with $H_{S}$ being the angular momentum operator. The optimal sensitivity is usually limited by the strength of noise in the system. Instead of using the entire system to probe the signal, one could prepare an encoded probe state using covariant codes where $H_{S}$ is mapped to $H_{L}$ associated with the logical system. Covariant codes with low infidelity significantly reduce the noise in the logical system and therefore provide a good sensitivity of the signal.

No-go theorems in quantum metrology [28-34] prevent the existence of perfectly error-correcting covariant codes in the above scenario. In particular, it was known that given a noise channel $\mathcal{N}_{S}(\cdot)=\sum_{i=1}^{r} K_{S, i}(\cdot) K_{S, i}^{\dagger}$ and a physical Hamiltonian $e^{-i H_{S} \theta}$, there exists an encoding channel $\mathcal{E}_{S \leftarrow L}$ and a recovery channel $\mathcal{R}_{L \leftarrow S}$ such that

$$
\mathcal{R}_{L \leftarrow S} \circ \mathcal{N}_{S} \circ \mathcal{U}_{S, \theta} \circ \mathcal{E}_{S \leftarrow L}
$$

is a non-trivial unitary channel only if $H_{S} \notin \operatorname{span}\left\{K_{S, i}^{\dagger} K_{S, j}, \forall i, j\right\}$ [34]. However, the above channel (Eq. (7)) with respect to any perfectly error-correcting covariant code is simply $\mathcal{U}_{L, \theta}$. Therefore, we conclude that perfectly error-correcting covariant codes do not exist when

$$
H_{S} \in \operatorname{span}\left\{K_{S, i}^{\dagger} K_{S, j}, \forall i, j\right\}
$$

which we call the "Hamiltonian-in-Kraus-span" (HKS) condition. One could check that local Hamiltonians with non-trivial local errors is a special case of the HKS condition.

\footnotetext{
${ }^{1}$ There are other equivalent definitions of the code infidelity in the literature that have a quadratic difference in terms of scaling with ours, e.g., $\sqrt{1-f^{2}}$ in [10] or $\sqrt{1-f}$ in [47].
} 
Note that the no-go result might be circumvented when the system dimension is infinite. To be more specific, perfect error-correcting codes that are covariant under $U(1)$ or even an arbitrary group $G$ can be constructed using quantum systems that transform as the regular representation of $G$ which are infinite-dimensional when $G$ is infinite and are equivalent to the notions of idealized clocks or perfect reference frames [9, 10, 12].

\subsection{Quantum channel estimation}

From the discussion above, we saw that no-go theorems in quantum metrology help us extend the scope of the Eastin-Knill theorem for covariant codes. As we will see below, a powerful lower bound for the infidelity of covariant codes could also be derived thanks to recent developments in quantum channel estimation [29, 30, 34-38].

Here we first review the definitions of QFIs for quantum states and then introduce the extensions to quantum channels. The QFI is a good measure of the amount of information a quantum state $\rho_{\theta}$ carries about an unknown parameter $\theta$, characterized by the the quantum Cramér-Rao bound [5861], $\delta \theta \geq 1 / \sqrt{N_{\operatorname{expr}} F\left(\rho_{\theta}\right)}$, where $\delta \theta$ is the standard deviation of any unbiased estimator of $\theta$, $N_{\text {expr }}$ is the number of repeated experiments and $F\left(\rho_{\theta}\right)$ is the QFI of $\rho_{\theta}$. The QFI as the quantum generalization of the classical Fisher information is not unique, due to the noncommutativity of quantum operators. Two most commonly used QFIs are the symmetric logarithmic derivative (SLD) QFI and the right logarithmic derivative (RLD) QFI, respectively defined by [58, 59, 62],

$$
\begin{gathered}
F_{\mathscr{S}}\left(\rho_{\theta}\right)=\operatorname{Tr}\left(\rho_{\theta}\left(L_{\theta}^{\mathscr{S}}\right)^{2}\right), \quad \partial_{\theta} \rho_{\theta}=\frac{1}{2}\left(L_{\theta}^{\mathscr{S}} \rho_{\theta}+\rho_{\theta} L_{\theta}^{\mathscr{S}}\right), \\
F_{\mathscr{R}}\left(\rho_{\theta}\right)=\operatorname{Tr}\left(\rho_{\theta} L_{\theta}^{\mathscr{R}} L_{\theta}^{\mathscr{R} \dagger}\right), \quad \partial_{\theta} \rho_{\theta}=\rho_{\theta} L_{\theta}^{\mathscr{R}},
\end{gathered}
$$

where the $\operatorname{SLD} L_{\theta}^{\mathscr{S}}$ is Hermitian and the $\operatorname{RLD} L_{\theta}^{\mathscr{R}}$ is linear. Note that $F_{\mathscr{R}}\left(\rho_{\theta}\right)=+\infty$ if $\operatorname{supp}\left(\partial_{\theta} \rho_{\theta}\right) \nsubseteq \operatorname{supp}\left(\rho_{\theta}\right)$. The QFIs satisfy many nice information-theoretic properties [38], such as additivity $F\left(\rho_{\theta} \otimes \sigma_{\theta}\right)=F\left(\rho_{\theta}\right)+F\left(\sigma_{\theta}\right)$ and monotonicity $F\left(\mathcal{N}\left(\rho_{\theta}\right)\right) \leq F\left(\rho_{\theta}\right)$ for $\theta$-independent channel $\mathcal{N}$. Note that the SLD QFI is the smallest monotonic quantum extension from the classical Fisher information and the quantum Cramér-Rao bound with respect to the SLD QFI is saturable asymptotically $\left(N_{\text {expr }} \gg 1\right)$.

In this section, we will focus on the SLD QFI for quantum channels. Discussions on the RLD QFI for quantum channels will be delayed to Sec. 4 where it is used. Given a quantum channel $\mathcal{N}_{\theta}$, the (entanglement-assisted) SLD QFI of $\mathcal{N}_{\theta}[35]$ is defined by

$$
F_{\mathscr{S}}\left(\mathcal{N}_{\theta}\right)=\max _{\rho} F_{\mathscr{S}}\left(\left(\mathcal{N}_{\theta} \otimes \mathbb{1}_{R}\right)(\rho)\right)
$$

where $R$ is an unbounded reference system. The regularized SLD QFI for quantum channels also has a single-letter expression [34]:

$$
\begin{aligned}
& F_{\mathscr{S}}^{\mathrm{reg}}\left(\mathcal{N}_{\theta}\right)=\lim _{N \rightarrow \infty} \frac{F_{\mathscr{S}}\left(\mathcal{N}_{\theta}^{\otimes N}\right)}{N}= \begin{cases}4 \min _{h: \beta_{\theta}=0}\left\|\alpha_{\theta}\right\| & (\mathrm{S}), \\
+\infty & \text { otherwise, }\end{cases} \\
& (\mathrm{S}): i \sum_{i=1}^{r} K_{i, \theta}^{\dagger} \partial_{\theta} K_{i, \theta} \in \operatorname{span}\left\{K_{i, \theta}^{\dagger} K_{j, \theta}, \forall i, j\right\}
\end{aligned}
$$

where $\mathcal{N}_{\theta}(\cdot)=\sum_{i=1}^{r} K_{i, \theta}(\cdot) K_{i, \theta}^{\dagger}, h$ is a Hermitian operator in $\mathbb{C}^{r \times r},\|\cdot\|$ is the operator norm and

$$
\begin{aligned}
\alpha_{\theta} & =\left(\partial_{\theta} \mathbf{K}_{\theta}+i h \mathbf{K}_{\theta}\right)^{\dagger}\left(\partial_{\theta} \mathbf{K}_{\theta}+i h \mathbf{K}_{\theta}\right), \\
\beta_{\theta} & =\mathbf{K}_{\theta}^{\dagger} h \mathbf{K}_{\theta}-i \mathbf{K}_{\theta}^{\dagger} \partial_{\theta} \mathbf{K}_{\theta} .
\end{aligned}
$$

Here $\mathbf{K}_{\theta}^{T}=\left(\begin{array}{llll}K_{1, \theta}^{T} & K_{2, \theta}^{T} \cdots K_{r, \theta}^{T}\end{array}\right) \in \mathbb{C}^{d \times r d}$ is a block matrix where ${ }^{T}$ means the transpose of a matrix.

Note that when $(\mathrm{S})$ is violated, $F_{\mathscr{S}}^{\mathrm{reg}}\left(\mathcal{N}_{\theta}\right)=\infty$ because we will have $F_{\mathscr{S}}\left(\mathcal{N}_{\theta}^{\otimes N}\right) \propto N^{2}[34]$. The regularized SLD QFI is additive (see Appx. A) and could be calculated efficiently using semidefinite programs (SDP) [29]. It is also monotonic, satisfying $F_{\mathscr{S}}^{\mathrm{reg}}\left(\Phi_{1} \circ\left(\mathcal{N}_{\theta} \otimes \mathbb{1}\right) \circ \Phi_{2}\right) \leq F_{\mathscr{S}}^{\mathrm{reg}}\left(\mathcal{N}_{\theta}\right)$ where $\Phi_{1,2}$ are any parameter-independent channels, due to the monotonicity of the state QFI. 


\subsection{Metrological bound}

In order to derive a lower bound on the infidelity of covariant codes using the channel QFI, we note that the channel QFI provides an upper limit to the sensitivity of $\theta$ for $\mathcal{N}_{S, \theta}=\mathcal{N}_{S} \circ \mathcal{U}_{S, \theta}$, which cannot be broken using covariant QEC.

Concretely, we consider an encoding scheme based on covariant QEC, where the original system consists of the physical system $S$ and a noiseless ancillary qubit $A$ and the logical system is a twodimensional system $C$. Suppose the original system is subject to Hamiltonian evolution $e^{-i H_{S} \theta}$ and noise channel $\mathcal{N}_{S}$, then the logical system will be subject to a $Z$-rotation signal and a rotated dephasing noise (defined later). Specifically, the Hamiltonian evolution in $C$ is $e^{-i\left(\Delta H_{L}\right) Z_{C} \theta / 2}$ where $Z_{C}$ is the Pauli-Z operator and the rotated dephasing noise channel has a noise rate smaller than the code infidelity. The monotonicity of the channel QFI guarantees that the QFI of the logical system cannot surpass the QFI of the original system and thus leads to a lower bound on the code infidelity. The Hamiltonians and noises before and after the covariant encoding scheme are listed in Fig. 1c.

To roughly estimate the scaling of the lower bound in the small infidelity limit, consider $N$ logical qubits each under a unitary evolution $e^{-i\left(\Delta H_{L}\right) Z_{C} \theta / 2}$ with a noise rate $\varepsilon$. It is known that the SLD QFI of a noiseless $N$-qubit GHZ state is $\left(\Delta H_{L}\right)^{2} N^{2}[63]$. Taking $N=\Theta(1 / \varepsilon)$, the total noise can be bounded by a small constant, and the state SLD QFI per qubit is still roughly $\Theta\left(\left(\Delta H_{L}\right)^{2} N\right)=\Theta\left(\left(\Delta H_{L}\right)^{2} / \varepsilon\right)$, which is always no greater than the regularized channel SLD QFI $F_{\mathscr{S}}^{\mathrm{reg}}\left(\mathcal{N}_{S, \theta}\right)$ before QEC. Thus, $\varepsilon$ must be lower bounded by $\Theta\left(\left(\Delta H_{L}\right)^{2} / F_{\mathscr{S}}^{\mathrm{reg}}\left(\mathcal{N}_{S, \theta}\right)\right)$. In fact, using the regularized SLD QFI for quantum channels, we can prove the following theorem:

Theorem 1. Consider a covariant code $\mathcal{E}_{S \leftarrow L}$ under a noise channel $\mathcal{N}_{S}(\cdot)=\sum_{i=1}^{r} K_{S, i}(\cdot) K_{S, i}^{\dagger}$. If the HKS condition is satisfied, i.e.,

$$
H_{S} \in \operatorname{span}\left\{K_{S, i}^{\dagger} K_{S, j}, \forall i, j\right\}
$$

then $\varepsilon\left(\mathcal{N}_{S}, \mathcal{E}_{S \leftarrow L}\right)$ is lower bounded as follows,

$$
\varepsilon \geq \ell_{1}\left(\frac{\left(\Delta H_{L}\right)^{2}}{4 F_{\mathscr{S}}^{\mathrm{reg}}\left(\mathcal{N}_{S}, H_{S}\right)}\right)=\frac{\left(\Delta H_{L}\right)^{2}}{4 F_{\mathscr{S}}^{\mathrm{reg}}\left(\mathcal{N}_{S}, H_{S}\right)}+O\left(\left(\frac{\left(\Delta H_{L}\right)^{2}}{4 F_{\mathscr{S}}^{\mathrm{reg}}\left(\mathcal{N}_{S}, H_{S}\right)}\right)^{2}\right),
$$

where $\ell_{1}(x)=(1+4 x-\sqrt{1+4 x}) /(2(1+4 x))=x+O\left(x^{2}\right)$ is a monotonically increasing function and $F_{\mathscr{S}}^{\mathrm{reg}}\left(\mathcal{N}_{S}, H_{S}\right)$ is the regularized $S L D Q F I$ of $\mathcal{N}_{S, \theta}$. Specifically, $F_{\mathscr{S}}^{\mathrm{reg}}\left(\mathcal{N}_{S}, H_{S}\right)=4 \min _{h: \beta_{S}=0}\left\|\alpha_{S}\right\|$, $h$ is a Hermitian operator in $\mathbb{C}^{r \times r} . \alpha_{S}$ and $\beta_{S}$ are Hermitian operators acting on $S$ defined by $\alpha_{S}=\mathbf{K}_{S}^{\dagger} h^{2} \mathbf{K}_{S}-H_{S}^{2}$ and $\beta_{S}=\mathbf{K}_{S}^{\dagger} h \mathbf{K}_{S}-H_{S}$, where $\mathbf{K}^{T}=\left(K_{1}^{T} K_{2}^{T} \cdots K_{r}^{T}\right) \in \mathbb{C}^{d_{S} \times r d_{S}}$ is a block matrix.

Note that $\ell_{1}(x) \leq 1 / 2$ for all $x$ and the lower bound does provide useful information when $\varepsilon \geq 1 / 2$. We remark that, in principle, the regularized SLD QFI on the right-hand side of Eq. (16) could be replaced by any other type of QFIs because the SLD QFI is the smallest monotonic quantum extension from the classical Fisher information. However, the regularized SLD QFI will always lead to the tightest bound using our metrological approach, as we will see later.

\subsection{Proof of Theorem 1}

The main obstacle to proving Theorem 1 is to relate the infidelity of covariant codes to the QFI of the effective quantum channel in the logical system. Here we overcome this obstacle and provide a proof of Theorem 1 by employing entanglement-assisted QEC to reduce $\mathcal{N}_{S, \theta}$ to rotated dephasing channels (the composition of dephasing channels and Pauli-Z rotations) whose QFI has simple mathematical forms, and then connecting the noise rate of the rotated dephasing channels to the infidelity of the covariant codes (see Fig. 1).

Single-qubit rotated dephasing channels take the form

$$
\mathcal{D}_{p, \phi}(\rho)=(1-p) e^{-i \frac{\phi}{2} Z} \rho e^{i \frac{\phi}{2} Z}+p e^{-i \frac{\phi}{2} Z} Z \rho Z e^{i \frac{\phi}{2} Z}
$$




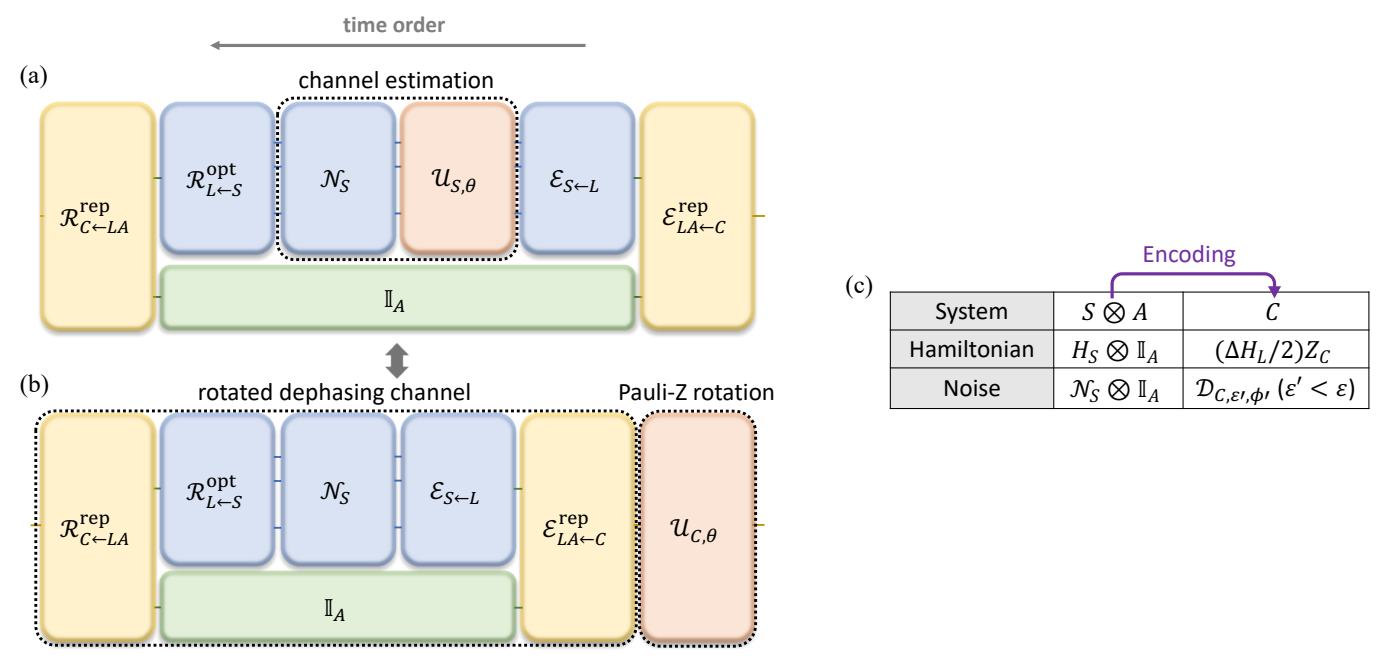

Figure 1: Reduction of $\mathcal{N}_{S, \theta}=\mathcal{N}_{S} \circ \mathcal{U}_{S, \theta}$ to rotated dephasing channels using entanglement-assisted QEC. (a) represents the quantum channel $\mathcal{R}_{C \leftarrow S A} \circ\left(\mathcal{N}_{S, \theta} \otimes \mathbb{1}_{A}\right) \circ \mathcal{E}_{S A \leftarrow C}$ with a channel QFI no larger than $F\left(\mathcal{N}_{S, \theta}\right)$. Because of the covariance of the code, (a) is equivalent to (b) which consists of a Pauli-Z rotation $\mathcal{U}_{C, \theta}$ and a $\theta$-independent rotated dephasing channel $\mathcal{I}_{C}$ whose noise rate is smaller than $\varepsilon\left(\mathcal{N}_{S}, \mathcal{E}_{S \leftarrow L}\right)$ (see Lemma 1 ). The Hamiltonians and noises before and after the encoding are listed in (c).

where $Z$ is the Pauli-Z operator, $0<p<1 / 2$ and $\phi$ is real. When $\phi$ is a function of $\theta$, we could calculate the regularized SLD QFI of $\mathcal{D}_{p, \phi_{\theta}}$ (see Appx. B in [34] or [64]):

$$
F_{\mathscr{S}}^{\mathrm{reg}}\left(\mathcal{D}_{p, \phi_{\theta}}\right)=\frac{(1-2 p)^{2}\left(\partial_{\theta} \phi_{\theta}\right)^{2}}{4 p(1-p)},
$$

which are both inversely proportional to the noise rate $p$ when $p$ is small - a crucial feature in deriving the lower bounds.

Next, we present an entanglement-assisted QEC protocol to reduce $\mathcal{N}_{S}$ to rotated dephasing channels with a noise rate lower than $\varepsilon\left(\mathcal{N}_{S}, \mathcal{E}_{S \leftarrow L}\right)$. Let $\left|0_{L}\right\rangle$ and $\left|1_{L}\right\rangle$ be eigenstates respectively corresponding to the largest and the smallest eigenvalues of $H_{L}$. Consider the following twodimensional entanglement-assisted code

$$
\mathcal{E}_{L A \leftarrow C}^{\mathrm{rep}}\left(\left|0_{C}\right\rangle\right)=\left|0_{L} 0_{A}\right\rangle, \quad \mathcal{E}_{L A \leftarrow C}^{\mathrm{rep}}\left(\left|1_{C}\right\rangle\right)=\left|1_{L} 1_{A}\right\rangle,
$$

where $A$ is a noiseless ancillary qubit and the superscript rep means "repetition". The encoding channel from the two-level system $C$ to $S A$ will simply be $\mathcal{E}_{S A \leftarrow C}=\left(\mathcal{E}_{S \leftarrow L} \otimes \mathbb{1}_{A}\right) \circ \mathcal{E}_{L A \leftarrow C}^{\text {rep }} \cdot \mathcal{E}_{S A \leftarrow C}$ is still a covariant code whose logical and physical Hamiltonians are

$$
H_{C}=\frac{\Delta H_{L}}{2} \cdot Z_{C}, \quad H_{S A}=H_{S} \otimes \mathbb{1}_{A} .
$$

The noiseless ancillary qubit will help us suppress off-diagonal noises in the system because any single qubit bit-flip noise on $L$ could be fully corrected by mapping $\left|i_{L} j_{A}\right\rangle$ to $\left|j_{C}\right\rangle$ for all $i, j$. In fact, $\mathcal{N}_{S}$ will be reduced to a rotated dephasing channel, as shown in the following lemma:

Lemma 1. Consider a noise channel $\mathcal{N}_{S A}=\mathcal{N}_{S} \otimes \mathbb{1}_{A}$. There exists a recovery channel $\mathcal{R}_{C \leftarrow S A}$ such that the effective noise channel $\mathcal{I}_{C}=\mathcal{R}_{C \leftarrow S A} \circ \mathcal{N}_{S A} \circ \mathcal{E}_{S A \leftarrow C}$ is a rotated dephasing channel, satisfying

$$
\mathcal{I}_{C}=\mathcal{D}_{C, \varepsilon^{\prime}, \phi^{\prime}},
$$

where $\varepsilon^{\prime} \leq \varepsilon\left(\mathcal{N}_{S}, \mathcal{E}_{S \leftarrow L}\right)$.

Proof. Consider the following recovery channel

$$
\mathcal{R}_{C \leftarrow S A}=\mathcal{R}_{C \leftarrow L A}^{\mathrm{rep}} \circ\left(\mathcal{R}_{L \leftarrow S}^{\mathrm{opt}} \otimes \mathbb{1}_{A}\right),
$$


where

$$
\begin{aligned}
\mathcal{R}_{C \leftarrow L A}^{\text {rep }}(\cdot)= & \left(\left|0_{C}\right\rangle\left\langle 0_{L} 0_{A}|+| 1_{C}\right\rangle\left\langle 1_{L} 1_{A}\right|\right)(\cdot)\left(\left|0_{L} 0_{A}\right\rangle\left\langle 0_{C}|+| 1_{L} 1_{A}\right\rangle\left\langle 1_{C}\right|\right)+ \\
& \left(\left|0_{C}\right\rangle\left\langle 1_{L} 0_{A}|+| 1_{C}\right\rangle\left\langle 0_{L} 1_{A}\right|\right)(\cdot)\left(\left|1_{L} 0_{A}\right\rangle\left\langle 0_{C}|+| 0_{L} 1_{A}\right\rangle\left\langle 1_{C}\right|\right)+ \\
& \sum_{i=2}^{d_{L}-1}\left(\left|0_{C}\right\rangle\left\langle i_{L} 0_{A}|+| 1_{C}\right\rangle\left\langle i_{L} 1_{A}\right|\right)(\cdot)\left(\left|i_{L} 0_{A}\right\rangle\left\langle 0_{C}|+| i_{L} 1_{A}\right\rangle\left\langle 1_{C}\right|\right) .
\end{aligned}
$$

The last term above disappears when $d_{L}=2$.

One could check that

$$
\mathcal{I}_{C}\left(\left|k_{C}\right\rangle\left\langle j_{C}\right|\right)= \begin{cases}\left|k_{C}\right\rangle\left\langle j_{C}\right|, & k=j, \\ \left(1-2 \varepsilon^{\prime}\right) e^{i \phi^{\prime}(k-j)}\left|k_{C}\right\rangle\left\langle j_{C}\right|, & k \neq j,\end{cases}
$$

which indicates that $\mathcal{I}_{C}=\mathcal{D}_{C, \varepsilon^{\prime}, \phi^{\prime}}$ (Eq. (21)). Here,

$$
\varepsilon^{\prime} \leq 1-f^{2}\left(\mathcal{I}_{C}, \mathbb{1}_{C}\right) \leq 1-f^{2}\left(\mathcal{I}_{L}^{\mathrm{opt}}, \mathbb{1}_{L}\right)=\varepsilon\left(\mathcal{N}_{S}, \mathcal{E}_{S \leftarrow L}\right) .
$$

where $\mathcal{I}_{L}=\mathcal{R}_{L \leftarrow S}^{\mathrm{opt}} \circ \mathcal{N}_{S} \circ \mathcal{E}_{S \leftarrow L}$ the first inequality follows from the worst-case entanglement fidelity for rotated dephasing channels (see Appx. B), and the the second inequality follows from $\mathbb{1}_{C}=\mathcal{R}_{C \leftarrow L A}^{\mathrm{rep}} \circ \mathcal{E}_{L A \leftarrow C}^{\mathrm{rep}}$ and the monotonicity of the fidelity [1].

Lemma 1 shows that $\mathcal{N}_{S}$ could be reduced to a rotated dephasing channel $\mathcal{I}_{C}$ through entanglement-assisted QEC. Consider parameter estimation of $\theta$ in the quantum channel $\mathcal{N}_{S, \theta}=$ $\mathcal{N}_{S} \circ \mathcal{U}_{S, \theta}$. We have the error-corrected quantum channel

$$
\mathcal{N}_{C, \theta}=\mathcal{R}_{C \leftarrow S A} \circ\left(\mathcal{N}_{S, \theta} \otimes \mathbb{1}_{A}\right) \circ \mathcal{E}_{S A \leftarrow C}=\mathcal{I}_{C} \circ \mathcal{U}_{C, \theta},
$$

equal to a rotated dephasing channel with noise rate $\varepsilon^{\prime}$ and phase $\phi_{\theta}=\phi^{\prime}+\Delta H_{L} \theta$. The monotonicity of the regularized channel SLD QFI implies that

$$
F_{\mathscr{S}}^{\mathrm{reg}}\left(\mathcal{N}_{S, \theta}\right) \geq F_{\mathscr{S}}^{\mathrm{reg}}\left(\mathcal{N}_{C, \theta}\right)
$$

where

$$
F_{\mathscr{S}}^{\mathrm{reg}}\left(\mathcal{N}_{S, \theta}\right)= \begin{cases}F_{\mathscr{S}}^{\mathrm{reg}}\left(\mathcal{N}_{S}, H_{S}\right) & H_{S} \in \operatorname{span}\left\{K_{S, i}^{\dagger} K_{S, j}, \forall i, j\right\} \\ +\infty & \text { otherwise }\end{cases}
$$

and

$$
F_{\mathscr{S}}^{\mathrm{reg}}\left(\mathcal{N}_{C, \theta}\right)=\frac{\left(1-2 \varepsilon^{\prime}\right)^{2}\left(\Delta H_{L}\right)^{2}}{4 \varepsilon^{\prime}\left(1-\varepsilon^{\prime}\right)} .
$$

Using Eq. (27) and $\varepsilon^{\prime} \leq \varepsilon<1 / 2$, we have

$$
\varepsilon \cdot \frac{1-\varepsilon}{(1-2 \varepsilon)^{2}} \geq \frac{\left(\Delta H_{L}\right)^{2}}{4 F_{\mathscr{S}}^{\mathrm{reg}}\left(\mathcal{N}_{S}, H_{S}\right)} .
$$

Theorem 1 then follows from the fact that $\ell_{1}(\cdot)$ is the inverse function of $x=\frac{\varepsilon(1-\varepsilon)}{(1-2 \varepsilon)^{2}}$ for $\varepsilon \in[0,1 / 2)$. Moreover, any other types of regularized monotonic QFIs of $\mathcal{D}_{p, \phi_{\theta}}$ have the same values as the regularized SLD QFI of $\mathcal{D}_{p, \phi_{\theta}}$ [64]. It implies that although the definition of QFI by generalizing the classical Fisher information is not unique, one cannot derive tighter bounds on the code infidelity by replacing the regularized SLD QFI with other types of QFIs in this proof.

\section{Resource-theoretic bound}

Now we demonstrate how quantum resource theory provides another pathway towards characterizing the limitations of covariant QEC. More specifically, the covariance property of the allowed operations indicates close connections to the (highly relevant) resource theories of asymmetry, reference frames, coherence, and quantum clocks [39-41, 44, 65]. In our current context, we work with a resource theory of coherence (see e.g., [44] for more discussions on the setting) where the 
free (incoherent) states are those with density operators commuting with the physical Hamiltonian $H_{S}$, and the free operations are covariant operations $\mathcal{C}_{L \leftarrow S}$ from $S$ to $L$ satisfying

$$
\mathcal{C}_{L \leftarrow S} \circ \mathcal{U}_{S, \theta}=\mathcal{U}_{L, \theta} \circ \mathcal{C}_{L \leftarrow S}, \forall \theta \in \mathbb{R}
$$

The free (covariant) operations are incoherence-preserving, i.e., map incoherent states to incoherent states even with the assistance of reference systems [44]. (Note that in [44] covariant operations are called time-translation invariant operations.)

The following lemma shows that the recovery channel $\mathcal{R}_{L \leftarrow S}$ for a covariant code can be assumed to be covariant under two conditions: (1) the noise channel and the symmetry transformation commutes (e.g., satisfied by the erasure and depolarizing channels of interest here); $(2) U_{S}(\theta)=$ $e^{-i H_{S} \theta}$ and $U_{L}(\theta)=e^{-i H_{L} \theta}$ share a common period $\tau$ (which is not necessarily the fundamental period), i.e., $U_{S, L}(\tau)=\mathbb{1}_{S, L}$. For example, when $U_{S}(\theta)$ and $U_{L}(\theta)$ are both representations of $U(1), \tau=2 \pi$ is the common period, which is a standard assumption in the theory of quantum clocks $[12,44]$.

Lemma 2. Suppose $\mathcal{N}_{S} \circ \mathcal{U}_{S, \theta}=\mathcal{U}_{S, \theta} \circ \mathcal{N}_{S}$ and $U_{L, S}(\theta)$ share a common period $\tau$. Then the code infidelity $\varepsilon\left(\mathcal{N}_{S}, \mathcal{E}_{S \leftarrow L}\right)$ and the Choi code infidelity $\varepsilon_{\text {Choi }}\left(\mathcal{N}_{S}, \mathcal{E}_{S \leftarrow L}\right)$ stay the same if the recovery channels are restricted to be covariant operations.

Proof. Let $\mathcal{R}_{L \leftarrow S}^{\mathrm{opt}}$ be the recovery channel such that $1-f^{2}\left(\mathcal{R}_{S \leftarrow L}^{\mathrm{opt}} \circ \mathcal{N}_{S} \circ \mathcal{E}_{S \leftarrow L}, \mathbb{1}_{L}\right)=\varepsilon\left(\mathcal{N}_{S}, \mathcal{E}_{S \leftarrow L}\right)$. Consider the following recovery channel:

$$
\mathcal{R}_{L \leftarrow S}^{\mathrm{cov}}=\frac{1}{\tau} \int_{0}^{\tau} d \theta \mathcal{U}_{L, \theta} \circ \mathcal{R}_{L \leftarrow S}^{\mathrm{opt}} \circ \mathcal{U}_{S, \theta}^{\dagger}
$$

We first observe that $\mathcal{R}_{L \leftarrow S}^{\text {cov }}$ is covariant:

$$
\mathcal{R}_{L \leftarrow S}^{\mathrm{cov}} \circ \mathcal{U}_{S, \theta^{\prime}}=\frac{1}{\tau} \int_{0}^{\tau} d \theta \mathcal{U}_{L, \theta} \circ \mathcal{R}_{L \leftarrow S}^{\mathrm{opt}} \circ \mathcal{U}_{S, \theta-\theta^{\prime}}^{\dagger}=\mathcal{U}_{L, \theta^{\prime}} \circ \mathcal{R}_{L \leftarrow S}^{\mathrm{cov}} .
$$

Furthermore,

$$
\mathcal{R}_{L \leftarrow S}^{\mathrm{cov}} \circ \mathcal{N}_{S} \circ \mathcal{E}_{S \leftarrow L}=\frac{1}{\tau} \int_{0}^{\tau} d \theta \mathcal{U}_{L, \theta} \circ\left(\mathcal{R}_{L \leftarrow S}^{\mathrm{opt}} \circ \mathcal{N}_{S} \circ \mathcal{E}_{S \leftarrow L}\right) \circ \mathcal{U}_{L, \theta}^{\dagger},
$$

Using the concavity of $f^{2}(\Phi, \mathbb{1})$ with respect to $\Phi$ and the monotonicity of the worst-case fidelity [45], we have $1-f^{2}\left(\mathcal{R}_{S \leftarrow L}^{\text {cov }} \circ \mathcal{N}_{S} \circ \mathcal{E}_{S \leftarrow L}, \mathbb{1}_{L}\right) \leq \varepsilon\left(\mathcal{N}_{S}, \mathcal{E}_{S \leftarrow L}\right)$. By definition, the equality holds.

Similarly, one could construct an optimal covariant recovery channel with respect to the Choi code infidelity by replacing $\mathcal{R}_{L \leftarrow S}^{\mathrm{opt}}$ with the optimal recovery channel achieving the minimum Choi infidelity in Eq. (32) and prove its optimality by noting that $f^{2}(\Phi, \mathbb{1})$ is linear with respect to the channel $\Phi$ and $f(\Phi, \mathbb{1})=f\left(\mathcal{U} \circ \Phi \circ \mathcal{U}^{\dagger}, \mathbb{1}\right)$ for any unitary channel $\mathcal{U}$.

This lemma allows us to formulate covariant QEC as a resource state conversion task, where one aims to transform noisy physical states to logical states by covariant operations (see Fig. 2a). It has recently been found that, by analyzing suitable resource monotones (functions of states that are nonincreasing under free operations), one can prove strong lower bounds on the infidelity of transforming generic noisy states to pure resource states by any free operation, which underlies the important task of distillation (see [42, 66, 67] for general results that apply to any well-behaved resource theory, and [44] for discussions specific to covariant operations). Here, we use the RLD QFI for quantum states, which is studied as a coherence monotone in [44], to derive bounds on the performance of covariant QEC. In particular, the RLD QFI satisfies

$$
F_{\mathscr{R}}\left(\mathcal{R}_{L \leftarrow S}^{\mathrm{cov}}\left(\rho_{S}\right), H_{L}\right) \leq F_{\mathscr{R}}\left(\rho_{S}, H_{S}\right),
$$

for all $\rho_{S}$ and covariant operations $\mathcal{R}_{L \leftarrow S}^{\text {cov }}$ where

$$
F_{\mathscr{R}}(\rho, H)=F_{\mathscr{R}}\left(e^{-i H \theta} \rho e^{i H \theta}\right)= \begin{cases}\operatorname{Tr}\left(H \rho^{2} H \rho^{-1}\right)-\operatorname{Tr}\left(\rho H^{2}\right) & \operatorname{supp}(H \rho H) \subseteq \operatorname{supp}(\rho) \\ +\infty & \text { otherwise }\end{cases}
$$


(a)
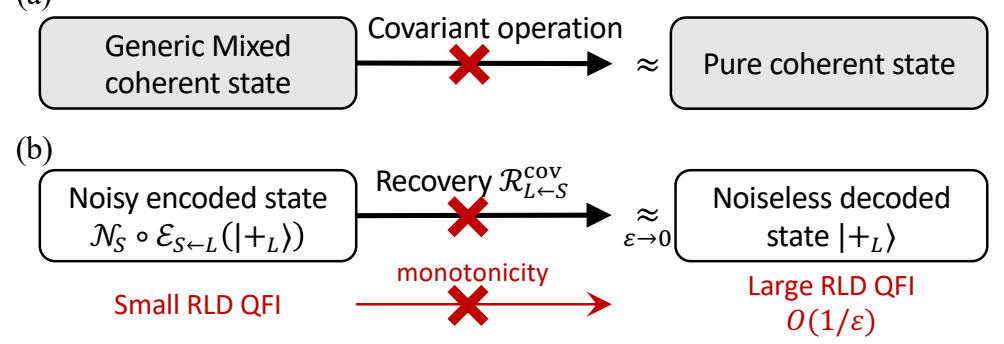

Figure 2: (a) As a case of the general no-go theorems for quantum resource purification, generic noisy coherent states cannot be transformed to states within a neighborhood of pure coherent states using covariant operations. (b) Specifically for covariant quantum error correction, a noisy coherent encoded state (e.g. $\left.\mathcal{N}_{S} \circ \mathcal{E}_{S \leftarrow L}\left(\left|+{ }_{L}\right\rangle\right)\right)$ cannot be transformed to states within a neighborhood of a pure coherent logical state (e.g. $\left.\left|+{ }_{L}\right\rangle\right)$, that is, there exist fundamental limits on the overall accuracy of recovery (decoding). As illustrated at the bottom, our specific bounds on the accuracy are derived from the monotonicity of RLD QFI under covariant operations. The key intuition is that RLD QFI diverges as the infidelity with the target pure coherent state $\varepsilon$ tends to zero, thus recovery with too small $\varepsilon$ is ruled out by the monotonicity of RLD QFI.

Notice that the RLD QFI $F_{\mathscr{R}}(\rho, H)$ approaches infinity when $\rho$ is coherent and its purity $\operatorname{Tr}\left(\rho^{2}\right)$ approaches one. Let $\rho_{S}=\mathcal{N}_{S} \circ \mathcal{E}_{S \leftarrow L}\left(\rho_{L}\right)$ where $\rho_{L}$ is a pure coherent state. If the right-hand side of Eq. (35) is finite, the left-hand side of Eq. (35) must also be finite, and thus a perfectly errorcorrecting recovery channel does not exist (see Fig. 2b). That is, the RLD QFI is a distinguished coherence monotone which can rule out generic noisy-to-pure transformations and further induce lower bounds on the code infidelity. In fact, the RLD QFI $F_{\mathscr{R}}(\rho, H)$ is lower bounded by $O(1 / \varepsilon)$ when $\rho$ is $\varepsilon$-close to a pure state $|\Psi\rangle$ in terms of infidelity, characterized by the following lemma:

Lemma 3 ([44]). Let $\tilde{\varepsilon}=1-\langle\Psi|\rho| \Psi\rangle$.

$$
F_{\mathscr{R}}(\rho, H) \geq\left(V_{H}(\Psi)-\frac{3 \sqrt{2 \tilde{\varepsilon}}(\Delta H)^{2}}{2}\right) \cdot \frac{1-3 \tilde{\varepsilon}+\tilde{\varepsilon}^{2}}{\tilde{\varepsilon}},
$$

where $V_{H}(\Psi)=\left\langle\Psi\left|H^{2}\right| \Psi\right\rangle-(\langle\Psi|H| \Psi\rangle)^{2}$.

Proof. According to Corollary 1 in Supplementary Note 3 in [44],

$$
F_{\mathscr{R}}(\rho, H) \geq V_{H}(\tilde{\Psi}) \cdot \frac{1-3 \tilde{\varepsilon}+\tilde{\varepsilon}^{2}}{\tilde{\varepsilon}}
$$

where $\tilde{\Psi}$ is the eigenvector of $\rho$ which corresponds to the largest eigenvalues of $\rho$. Let $\lambda_{\max }$ be the largest eigenvalue of $\rho$. Then $\langle\Psi|\rho| \Psi\rangle=1-\tilde{\varepsilon} \leq \lambda_{\max }$. Moreover,

$$
\begin{aligned}
1-\tilde{\varepsilon} & =\langle\Psi|\rho| \Psi\rangle \leq\left\langle\Psi\left|\left(\lambda_{\max }|\tilde{\Psi}\rangle\left\langle\tilde{\Psi}\left|+\rho-\lambda_{\max }\right| \tilde{\Psi}\right\rangle\langle\tilde{\Psi}|\right)\right| \Psi\right\rangle \\
& \leq \lambda_{\max }|\langle\Psi \mid \tilde{\Psi}\rangle|^{2}+\left(1-\lambda_{\max }\right)\left(1-|\langle\Psi \mid \tilde{\Psi}\rangle|^{2}\right) \leq \lambda_{\max }|\langle\Psi \mid \tilde{\Psi}\rangle|^{2}+\tilde{\varepsilon}\left(1-|\langle\Psi \mid \tilde{\Psi}\rangle|^{2}\right),
\end{aligned}
$$

leading to $|\langle\Psi \mid \tilde{\Psi}\rangle|^{2} \geq 1-2 \tilde{\varepsilon}$. Without loss of generality, assume the largest and the smallest eigenvalues of $H$ is $\frac{\Delta H}{2}$ and $-\frac{\Delta H}{2}$. Then

$$
\begin{aligned}
\left|V_{H}(\tilde{\Psi})-V_{H}(\Psi)\right| \leq & \operatorname{Tr}(H(|\tilde{\Psi}\rangle\langle\tilde{\Psi}|-| \Psi\rangle\langle\Psi|) H|\tilde{\Psi}\rangle\langle\tilde{\Psi}|) \mid \\
& +\mid \operatorname{Tr}\left((|\tilde{\Psi}\rangle\langle\tilde{\Psi}|-| \Psi\rangle\langle\Psi|)\left(H^{2}-H|\tilde{\Psi}\rangle\langle\tilde{\Psi}| H\right) \mid\right. \\
\leq & \||\tilde{\Psi}\rangle\langle\tilde{\Psi}|-| \Psi\rangle\langle\Psi| \|_{1}\left(\left\langle\Psi\left|H^{2}\right| \Psi\right\rangle+\| H^{2}-H|\tilde{\Psi}\rangle\langle\tilde{\Psi}| H \|\right) \\
\leq & 2 \sqrt{2 \tilde{\varepsilon}} \cdot \frac{3(\Delta H)^{2}}{4},
\end{aligned}
$$

where in the second step we use $|\operatorname{Tr}(A B)| \leq\|A\|_{1}\|B\|$ and in the third step we use $\||\tilde{\Psi}\rangle\langle\tilde{\Psi}|-| \Psi\rangle\langle\Psi| \|_{1}=2 \sqrt{1-|\langle\Psi \mid \tilde{\Psi}\rangle|^{2}} \leq 2 \sqrt{2 \tilde{\varepsilon}}$. Eq. (37) then follows from Eq. (38) and Eq. (40). 
Given Lemma 2 and Lemma 3, we are now ready to present our resource-theoretic bound on the code infidelity. The intuition on the operational level is that the accuracy of the optimal recovery transformation, which can be understood as a resource conversion task, is limited by the monotonicity of RLD QFI that diverges as approaching the pure target state (see Fig. 2b). More specifically, we compare the resource monotone, i.e., the RLD QFI of the decoded coherent state with that of the original noisy coherent state. The lower bound then follows from the monotonicity of the RLD QFI and the fact that the RLD QFI of the decoded coherent state is lower bounded by $O(1 / \varepsilon)$.

Note that we will also use the RLD QFI for quantum channels defined by $[36,38]$

$$
F_{\mathscr{R}}\left(\mathcal{N}_{\theta}\right)= \begin{cases}\left\|\operatorname{Tr}_{S\left(\mathcal{N}_{\theta}\right)}\left(\left(\partial_{\theta} \Gamma^{\mathcal{N}_{\theta}}\right)\left(\Gamma^{\mathcal{N}_{\theta}}\right)^{-1}\left(\partial_{\theta} \Gamma^{\mathcal{N}_{\theta}}\right)\right)\right\| & (\mathrm{R}) \\ +\infty & \text { otherwise }\end{cases}
$$

$(\mathrm{R}): \operatorname{span}\left\{\partial_{\theta} K_{i, \theta}, \forall i\right\} \subseteq \operatorname{span}\left\{K_{i, \theta}, \forall i\right\}$,

where $\mathcal{N}_{\theta}(\cdot)=\sum_{i=1}^{r} K_{i, \theta}(\cdot) K_{i, \theta}^{\dagger}$. Here we use the Choi operator of $\mathcal{N}_{\theta}: \Gamma^{\mathcal{N}_{\theta}}=\left(\mathcal{N}_{\theta} \otimes \mathbb{1}\right)(\Gamma)$, where $\Gamma=|\Gamma\rangle\langle\Gamma|$ and $|\Gamma\rangle=\sum_{i}|i\rangle|i\rangle . S\left(\mathcal{N}_{\theta}\right)$ denotes the output system of $\mathcal{N}_{\theta} . F_{\mathscr{R}}\left(\mathcal{N}_{\theta}\right)$ is also additive [36]. The result we obtained is the following.

Theorem 2. Consider a covariant code $\mathcal{E}_{S \leftarrow L}$ under a noise channel $\mathcal{N}_{S}(\cdot)=\sum_{i=1}^{r} K_{S, i}(\cdot) K_{S, i}^{\dagger}$. If $\mathcal{N}_{S}$ commutes with $\mathcal{U}_{S, \theta}, U_{L, S}(\theta)$ share a common period and

$$
\operatorname{span}\left\{K_{S, i} H_{S}, \forall i\right\} \subseteq \operatorname{span}\left\{K_{S, i}, \forall i\right\},
$$

then $\varepsilon$ and $\varepsilon_{\text {Choi }}$ are lower bounded as follows,

$$
\varepsilon \geq \ell_{2}\left(\frac{\left(\Delta H_{L}\right)^{2}}{4 F_{\mathscr{R}}\left(\mathcal{N}_{S}, H_{S}\right)}\right)=\frac{\left(\Delta H_{L}\right)^{2}}{4 F_{\mathscr{R}}\left(\mathcal{N}_{S}, H_{S}\right)}+O\left(\left(\frac{\left(\Delta H_{L}\right)^{2}}{4 F_{\mathscr{R}}\left(\mathcal{N}_{S}, H_{S}\right)}\right)^{2}\right),
$$

and

$$
\varepsilon_{\text {Choi }} \geq \ell_{3}\left(\frac{\operatorname{Tr}\left(H_{L}^{2}\right)}{d_{L} F_{\mathscr{R}}\left(\mathcal{N}_{S}, H_{S}\right)}\right)=\frac{\operatorname{Tr}\left(H_{L}^{2}\right)}{d_{L} F_{\mathscr{R}}\left(\mathcal{N}_{S}, H_{S}\right)}+O\left(\left(\frac{\operatorname{Tr}\left(H_{L}^{2}\right)}{d_{L} F_{\mathscr{R}}\left(\mathcal{N}_{S}, H_{S}\right)}\right)^{2}\right),
$$

where $\ell_{2}(x)=x+O\left(x^{2}\right)$ is the inverse function of the monotonic increasing function $x=$ $1 /\left(\left(1-3 \varepsilon+\varepsilon^{2}\right)(1-6 \sqrt{2 \varepsilon})\right)$ for $\varepsilon \in[0,1 / 72), \ell_{3}(x)=x+O\left(x^{2}\right)$ is the inverse function of the monotonic increasing function $x=1 /\left(\left(1-3 \varepsilon+\varepsilon^{2}\right)\left(1-\frac{3 d_{L}\left(\Delta H_{L}\right)^{2}}{2 \operatorname{Tr}\left(H_{L}^{2}\right)} \sqrt{2 \varepsilon}\right)\right)$ for $\varepsilon \in\left[0, \frac{2 \operatorname{Tr}\left(H_{L}^{2}\right)^{2}}{9 d_{L}^{2}\left(\Delta H_{L}\right)^{4}}\right)$, and $F_{\mathscr{R}}\left(\mathcal{N}_{S}, H_{S}\right)$ is the RLD QFI of $\mathcal{N}_{S, \theta}$. Specifically, $F_{\mathscr{R}}\left(\mathcal{N}_{S}, H_{S}\right)=\left\|\operatorname{Tr}_{S}\left(\Gamma_{S R}^{\mathcal{N}_{S}, H_{S}}\left(\Gamma_{S R}^{\mathcal{N}_{S}}\right)^{-1} \Gamma_{S R}^{\mathcal{N}_{S}, H_{S}}\right)\right\|$, with $\Gamma_{S R}^{\mathcal{N}_{S}}=\left(\mathcal{N}_{S} \otimes \mathbb{1}_{R}\right)\left(\Gamma_{S R}\right)$ and $\Gamma_{S R}^{\mathcal{N}_{S}, H_{S}}=\left(\mathcal{N}_{S} \otimes \mathbb{1}_{R}\right)\left(\left(H_{S} \otimes \mathbb{1}_{R}\right) \Gamma_{S R}\right)-\left(\mathcal{N}_{S} \otimes \mathbb{1}_{R}\right)\left(\Gamma_{S R}\left(H_{S} \otimes \mathbb{1}_{R}\right)\right)$, where $\Gamma_{S R}=|\Gamma\rangle_{S R}\left\langle\left.\Gamma\right|_{S R} \text { and } \mid \Gamma\right\rangle_{S R}=\sum_{i}|i\rangle_{S}|i\rangle_{R}$.

Proof. Let $\left|{ }_{L}\right\rangle=\frac{\left|0_{L}\right\rangle+\left|1_{L}\right\rangle}{\sqrt{2}}$. Then according to Lemma 2, there exists a covariant recovery channel $\mathcal{R}_{L \leftarrow S}^{\text {cov }}$ such that

$$
1-\left\langle{ }_{L}\left|\rho_{L}\right|+{ }_{L}\right\rangle \leq \varepsilon,
$$

where $\rho_{L}=\left(\mathcal{R}_{L \leftarrow S}^{\text {cov }} \circ \mathcal{N}_{S} \circ \mathcal{E}_{S \leftarrow L}\right)\left(\left|+{ }_{L}\right\rangle\left\langle{ }_{L}\right|\right)$. According to Lemma 3,

$$
F_{\mathscr{R}}\left(\rho_{L}, H_{L}\right) \geq \frac{1-3 \varepsilon+\varepsilon^{2}}{\varepsilon} \cdot\left(V_{H_{L}}\left(\left|+_{L}\right\rangle-\frac{3 \sqrt{2 \varepsilon}\left(\Delta H_{L}\right)^{2}}{2}\right),\right.
$$

where the variance $V_{H_{L}}\left(\left|+_{L}\right\rangle\right)=\left\langle+_{L}\left|H_{L}^{2}\right|+_{L}\right\rangle-\left\langle+_{L}\left|H_{L}\right|+_{L}\right\rangle^{2}=\frac{\left(\Delta H_{L}\right)^{2}}{4} . \varepsilon<1 / 72$ guarantees the right-hand side is positive. On the other hand, using Eq. (35),

$$
F_{\mathscr{R}}\left(\rho_{L}, H_{L}\right) \leq F_{\mathscr{R}}\left(\rho_{S}, H_{S}\right)=F_{\mathscr{R}}\left(\mathcal{N}_{S, \theta}\left(\mathcal{E}_{S \leftarrow L}\left(\left|+_{L}\right\rangle\left\langle{ }_{L}\right|\right)\right)\right) \leq F_{\mathscr{R}}\left(\mathcal{N}_{S, \theta}\right),
$$

where $\rho_{S}=\left(\mathcal{N}_{S} \circ \mathcal{E}_{S \leftarrow L}\right)\left(\left|+{ }_{L}\right\rangle\left\langle+_{L}\right|\right)$ and

$$
F_{\mathscr{R}}\left(\mathcal{N}_{S, \theta}\right)= \begin{cases}F_{\mathscr{R}}\left(\mathcal{N}_{S}, H_{S}\right) & \text { Eq. }(42) \\ +\infty & \text { otherwise }\end{cases}
$$


Using Eq. (46) and Eq. (47), we have

$$
\varepsilon \cdot \frac{1}{\left(1-3 \varepsilon+\varepsilon^{2}\right)(1-6 \sqrt{2 \varepsilon})} \geq \frac{\left(\Delta H_{L}\right)^{2}}{4 F_{\mathscr{R}}\left(\mathcal{N}_{S}, H_{S}\right)} .
$$

Eq. (43) then follows from the fact that $\ell_{2}(\cdot)$ is the inverse function of $x=\frac{1}{\left(1-3 \varepsilon+\varepsilon^{2}\right)(1-6 \sqrt{2 \varepsilon})}$.

Similarly, let $\left|\gamma_{L R}\right\rangle=\frac{1}{\sqrt{d_{L}}} \sum_{i=1}^{d_{L}}|i\rangle_{L}|i\rangle_{R}$. Then according to Lemma 2, there exists a covariant recovery channel $\mathcal{R}_{L \leftarrow S}^{\text {Choi }}$ such that

$$
1-\left\langle\gamma_{L R}\left|\rho_{L R}\right| \gamma_{L R}\right\rangle \leq \varepsilon_{\text {Choi }}
$$

According to Lemma 3,

$$
\begin{aligned}
F_{\mathscr{R}}\left(\rho_{L R}, H_{L} \otimes \mathbb{1}_{R}\right) & \geq \frac{1-3 \varepsilon+\varepsilon^{2}}{\varepsilon} \cdot\left(V_{H_{L} \otimes \mathbb{1}_{R}}\left(\left|\gamma_{L R}\right\rangle\right)-\frac{3 \sqrt{2 \varepsilon}\left(\Delta H_{L}\right)^{2}}{2}\right) \\
& \geq \frac{1-3 \varepsilon+\varepsilon^{2}}{\varepsilon} \cdot\left(\frac{\operatorname{Tr}\left(H_{L}^{2}\right)}{d_{L}}-\frac{3 \sqrt{2 \varepsilon}\left(\Delta H_{L}\right)^{2}}{2}\right) .
\end{aligned}
$$

The rest of the proof is exactly the same as in the proof of the lower bound on the worst-case infidelity.

As mentioned before, it is always true that $F_{\mathscr{R}}\left(\mathcal{N}_{S, \theta}\right) \geq F_{\mathscr{S}}^{\text {reg }}\left(\mathcal{N}_{S, \theta}\right)$. Moreoever, $\ell_{3}(x) \leq \ell_{2}(x) \leq$ $\ell_{1}(x)$ for all $x,{ }^{2}$ therefore Theorem 1 provides a tighter bound on the code infidelity than Theorem 2 . Note that the resource theory approach operates on the state level, which is fundamentally different from the metrological approach. The assumptions and quantities involved in the two approaches are also different. From the proof of Theorem 2, we see that there are three advantages of the resource-theoretic approach: (1) We can also derive a lower bound on the Choi code infidelity. (2) We can replace the entanglement-assisted RLD QFI with the one without entanglement assistance $\max _{\rho} F_{\mathscr{R}}\left(\mathcal{N}_{\theta}(\rho)\right)$ in Eq. (43) which might tighten it in certain scenarios. (3) The lower bounds are state-dependent, e.g., we are allowed to replace $F_{\mathscr{R}}\left(\mathcal{N}_{S}, H_{S}\right)$ with $F_{\mathscr{R}}\left(\left(\mathcal{N}_{S} \circ \mathcal{E}_{S \leftarrow L}\right)\left(\left|+{ }_{L}\right\rangle\left\langle{ }_{L}\right|\right), H_{S}\right)$ in Eq. (43), which may be of independent interest in determining the lower bounds on the code infidelity for some special types of covariant codes.

\section{Local Hamiltonians and local noises}

One of the most common scenarios where covariant codes are considered is when $S$ is an $n$-partite system, consisting of subsystems $S_{1}, S_{2}, \ldots, S_{n}$. The physical Hamiltonian and the noise channel are both local, given by

$$
H_{S}=\sum_{k=1}^{n} H_{S_{k}}, \quad \mathcal{N}_{S}=\bigotimes_{k=1}^{n} \mathcal{N}_{S_{k}}, \quad \mathcal{N}_{S_{k}}(\cdot)=\sum_{i=1}^{r_{k}} K_{S_{k}, i}(\cdot) K_{S_{k}, i}^{\dagger} .
$$

In general, it takes exponential time (in the number of subsystems) to solve our lower bounds on the code infidelity. However, when the Hamiltonians and the noises are local, using the additivity of channel QFIs, we could directly calculate the lower bounds, requiring only computation of the channel QFIs in each subsystem. To be specific, for $\varepsilon$-correctable codes under $\mathcal{N}_{S}$, Theorem 1 indicates that when

$$
H_{S_{k}} \in \operatorname{span}\left\{K_{S_{k}, i}^{\dagger} K_{S_{k}, j}, \forall i, j\right\}, \quad \forall k,
$$

we have

$$
\varepsilon \geq \ell_{1}\left(\frac{\left(\Delta H_{L}\right)^{2}}{4 \sum_{k=1}^{n} F_{\mathscr{S}}^{\mathrm{reg}}\left(\mathcal{N}_{S_{k}}, H_{S_{k}}\right)}\right)
$$

${ }^{2}$ Note that when $d_{L}=2, \ell_{2}(x)=\ell_{3}(x)$. 
Instead of finding bounds for local noise channels $\mathcal{N}_{S}$ with certain noise rates, we sometimes are more interested in the capability of a code to correct single errors (each described by $\mathcal{M}_{S_{k}}$ ). Consider the single-error noise channel

$$
\mathcal{M}_{S}=\sum_{k=1}^{n} q_{k} \mathcal{M}_{S_{k}}, \quad \sum_{k=1}^{n} q_{k}=1
$$

where $q_{k}$ is the probability that an error $\mathcal{M}_{S_{k}}$ occurs on the $k$-th subsystem. In order to obtain lower bounds on the code infidelity under noise channels $\mathcal{M}_{S}$, we use the following local noise channel

$$
\mathcal{N}_{S}(\delta)=\bigotimes_{k=1}^{n} \mathcal{N}_{S_{k}}(\delta)=\bigotimes_{k=1}^{n}\left(\left(1-\delta q_{k}\right) \mathbb{1}+\delta q_{k} \mathcal{M}_{S_{k}}\right)=(1-\delta) \mathbb{1}+\delta \sum_{k=1}^{n} q_{k} \mathcal{M}_{S_{k}}+O\left(\delta^{2}\right),
$$

whose local noise rates are proportional to a small positive parameter $\delta$. Using the concavity of $f^{2}(\Phi, \mathbb{1})$ with respect to the channel $\Phi$, we have

$$
f^{2}\left(\mathcal{R}_{L \leftarrow S} \circ \mathcal{N}_{S}(\delta) \circ \mathcal{E}_{S \leftarrow L}, \mathbb{1}_{L}\right) \geq(1-\delta)+\delta f^{2}\left(\mathcal{R}_{L \leftarrow S} \circ \mathcal{M}_{S} \circ \mathcal{E}_{S \leftarrow L}, \mathbb{1}_{L}\right)+O\left(\delta^{2}\right) .
$$

Taking the limit $\delta \rightarrow 0^{+}$, we must have $\varepsilon\left(\mathcal{M}_{S}, \mathcal{E}_{S \leftarrow L}\right) \geq \liminf \inf _{\delta \rightarrow 0^{+}} \frac{1}{\delta} \cdot \varepsilon\left(\mathcal{N}_{S}(\delta), \mathcal{E}_{S \leftarrow L}\right)$. Therefore, for $\varepsilon$-correctable codes under single-error noise channels $\mathcal{M}_{S}$, Theorem 1 indicates that when Eq. (53) is satisfied,

$$
\varepsilon \geq \liminf _{\delta \rightarrow 0^{+}} \ell_{1}\left(\frac{\left(\Delta H_{L}\right)^{2}}{4 \delta \sum_{k=1}^{n} F_{\mathscr{S}}^{\mathrm{reg}}\left(\mathcal{N}_{S_{k}}(\delta), H_{S_{k}}\right)}\right)
$$

Note that discussions here analogously apply to Theorem 2 due to the additivity of the channel RLD QFI, although we will only focus on Theorem 1 starting now since it provides the tightest bound in the following scenarios.

For generic error models with noises on multiple subsystems, e.g., the random phase error model in [12], the regularized SLD QFI and thus the lower bound on the code infidelity could be difficult to compute. In Appx. C, we derive an upper bound on the regularized SLD QFI for multierror noise channels which is efficiently computable in certain scenarios and provide an example of multiple erasure errors and local Hamiltonians.

\subsection{Erasure noise}

Now we present our bounds for the local erasure noise channel $\mathcal{N}^{\mathrm{e}}(\rho)=(1-p) \rho+p|\operatorname{vac}\rangle\langle\mathrm{vac}|$ on each subsystem. Here $p$ is the noise rate and we use the vacuum state $|v a c\rangle$ to represent the state of the erased subsystems. The Kraus operators for $\mathcal{N}^{\mathrm{e}}$ are

$$
K_{1}=\sqrt{1-p} \mathbb{1}, \quad K_{i+1}=\sqrt{p}|\operatorname{vac}\rangle\langle i|, \quad \forall 1 \leq i \leq d
$$

Different subsystems can have different noise rates $p_{k}$ and dimensions $d_{k}$. As derived in Appx. D, the regularized SLD QFI for erasure noise is

$$
F_{\mathscr{S}}^{\mathrm{reg}}\left(\mathcal{N}^{\mathrm{e}}, H\right)=(\Delta H)^{2} \frac{1-p}{p} .
$$

For $\varepsilon$-correctable codes under local erasure noise channel $\mathcal{N}_{S}^{\mathrm{e}}=\bigotimes_{k=1}^{n} \mathcal{N}_{S_{k}}^{\mathrm{e}}$, we have

$$
\varepsilon \geq \ell_{1}\left(\frac{\left(\Delta H_{L}\right)^{2}}{4 \sum_{k=1}^{n} \frac{1-p_{k}}{p_{k}}\left(\Delta H_{S_{k}}\right)^{2}}\right)
$$

using Eq. (54). For $\varepsilon$-correctable codes under single-error erasure noise channel $\mathcal{M}_{S}^{\mathrm{e}}=$ $\sum_{k=1}^{n} q_{k} \mathcal{M}_{S_{k}}^{\mathrm{e}}$ where $\mathcal{M}_{S_{k}}^{\mathrm{e}}\left(\rho_{S_{k}}\right)=|\operatorname{vac}\rangle\left\langle\left.\operatorname{vac}\right|_{S_{k}}\right.$,

$$
\varepsilon \geq \ell_{1}\left(\frac{\left(\Delta H_{L}\right)^{2}}{4 \sum_{k=1}^{n} \frac{1}{q_{k}}\left(\Delta H_{S_{k}}\right)^{2}}\right)
$$


using Eq. (58). In particular, when the probability of erasure is uniform on each subsystem, i.e., $q_{k}=\frac{1}{n}$, we have

$$
\varepsilon \geq \ell_{1}\left(\frac{\left(\Delta H_{L}\right)^{2}}{4 n \sum_{k=1}^{n}\left(\Delta H_{S_{k}}\right)^{2}}\right)=\frac{\left(\Delta H_{L}\right)^{2}}{4 n \sum_{k=1}^{n}\left(\Delta H_{S_{k}}\right)^{2}}+O\left(\left(\frac{\left(\Delta H_{L}\right)^{2}}{4 n \sum_{k=1}^{n}\left(\Delta H_{S_{k}}\right)^{2}}\right)^{2}\right) .
$$

As a comparison, Theorem 1 in [10] says that

$$
\varepsilon \geq \frac{\left(\Delta H_{L}\right)^{2}}{4 n^{2} \max _{k}\left(\Delta H_{S_{k}}\right)^{2}}
$$

Our bound Eq. (63) has a clear advantage in the small infidelity limit by improving the maximum of $\Delta H_{S_{k}}$ to their quadratic mean. A direct implication of Eq. (63) is an improved approximate Eastin-Knill theorem which establishes the infidelity lower bound for covariant codes with respect to special unitary groups (see Appx. F).

\subsection{Depolarizing noise}

Next, we present our bounds for local depolarizing noise channel $\mathcal{N}^{\mathrm{d}}(\rho)=(1-p) \rho+p \frac{\mathbb{1}}{d}$ on each subsystem, which has not been studied before. Again, we assume different subsystems can have different noise rates $p_{k}$ and dimensions $d_{k}$. The Kraus operators for $\mathcal{N}^{\mathrm{d}}$ are

$$
K_{1}=\sqrt{1-\frac{d^{2}-1}{d^{2}} p \mathbb{1}}, \quad K_{i}=\sqrt{\frac{p}{d^{2}}} U_{i-1}, \quad \forall 2 \leq i \leq d^{2},
$$

where $\left\{U_{0}=\mathbb{1}, U_{1}, \ldots, U_{d_{k}^{2}-1}\right\}$ is a unitary orthonormal basis in $\mathbb{C}^{d \times d}$.

In order to apply Theorem 1, we need to solve the following SDP

$$
F_{\mathscr{S}}^{\mathrm{reg}}\left(\mathcal{N}^{\mathrm{d}}, H\right)=\min _{h: \beta=0} 4\|\alpha\|,
$$

where $\beta=\mathbf{K}^{\dagger} h \mathbf{K}-H$ and $\alpha=\mathbf{K}^{\dagger} h^{2} \mathbf{K}-H^{2}$.

When $d=2$, as shown in Appx. D, we have $F_{\mathscr{S}}^{\mathrm{reg}}\left(\mathcal{N}^{\mathrm{d}}, H\right)=(\Delta H)^{2} \frac{2(1-p)^{2}}{p(3-2 p)}$. When all subsystems are qubits, for $\varepsilon$-correctable codes under local depolarizing noise channels $\mathcal{N}_{S}^{\mathrm{d}}=\bigotimes_{k=1}^{n} \mathcal{N}_{S_{k}}^{\mathrm{d}}$,

$$
\varepsilon \geq \ell_{1}\left(\frac{\left(\Delta H_{L}\right)^{2}}{4 \sum_{k=1}^{n} \frac{2\left(1-p_{k}\right)^{2}}{p_{k}\left(3-2 p_{k}\right)}\left(\Delta H_{S_{k}}\right)^{2}}\right)
$$

using Eq. (54) and for $\varepsilon$-correctable codes under single-error depolarizing noise channels $\mathcal{M}_{S}^{\mathrm{d}}=$ $\sum_{k=1}^{n} q_{k} \mathcal{M}_{S_{k}}^{\mathrm{d}}$ where $\mathcal{M}_{S_{k}}^{\mathrm{d}}\left(\rho_{S_{k}}\right)=\frac{\mathbb{1}}{2}$

$$
\varepsilon \geq \ell_{1}\left(\frac{3\left(\Delta H_{L}\right)^{2}}{8 \sum_{k=1}^{n} \frac{1}{q_{k}}\left(\Delta H_{S_{k}}\right)^{2}}\right)
$$

using Eq. (58).

The situation is more complicated when $d>2$, because the regularized SLD QFI may not have a closed-form expression. Instead, we show in Appx. E that

$$
F_{\mathscr{S}}^{\mathrm{reg}}\left(\mathcal{N}^{\mathrm{d}}, H\right) \leq(\Delta H)^{2} \frac{(1-p)^{2}}{p\left(1+\frac{2}{d^{2}}-p\right)} \leq(\Delta H)^{2} \frac{1-p}{p},
$$

by choosing a special $h$ which satisfies $\beta=0$ to calculate an upper bound on $4 \min _{h: \beta=0}\|\alpha\|$. Note that the right-hand side of Eq. (69) is equal to the regularized SLD QFI for erasure channels Eq. (60). We conclude that Eqs. (61)-(63) hold true for general depolarizing channels as well, regardless of the dimensions of subsystems. We also remark that the upper bound on the regularized SLD QFI for depolarizing channels we derived here might be of independent interest in quantum metrology. 
Note that the channel RLD QFI $F_{\mathscr{R}}\left(\mathcal{N}_{S_{k}}^{\mathrm{d}}, H_{S_{k}}\right)$ also upper bounds $F_{\mathscr{S}}^{\mathrm{reg}}\left(\mathcal{N}_{S_{k}}^{\mathrm{d}}, H_{S_{k}}\right)$ and has a closed-form expression. The RLD QFI $F_{\mathscr{R}}\left(\mathcal{N}^{\mathrm{d}}, H\right)$ for depolarizing channels could be directly calculated using

$$
F_{\mathscr{R}}\left(\mathcal{N}^{\mathrm{d}}, H\right)=\left\|\operatorname{Tr}_{S\left(\mathcal{N}^{\mathrm{d}}\right)}\left(\Gamma^{\mathcal{N}^{\mathrm{d}}, H}\left(\Gamma^{\mathcal{N}^{\mathrm{d}}}\right)^{-1} \Gamma^{\mathcal{N}^{\mathrm{d}}, H}\right)\right\|,
$$

where $\Gamma^{\mathcal{N}^{\mathrm{d}}}=\left(\mathcal{N}^{\mathrm{d}} \otimes \mathbb{1}\right) \Gamma, \Gamma^{\mathcal{N}^{\mathrm{d}}, H}=\left(\mathcal{N}^{\mathrm{d}} \otimes \mathbb{1}\right)(|H\rangle\langle\Gamma|-| \Gamma\rangle\langle H|)$ and $|H\rangle=(H \otimes \mathbb{1})|\Gamma\rangle$. Then

$$
F_{\mathscr{R}}\left(\mathcal{N}^{\mathrm{d}}, H\right)=\frac{(1-p)^{2}}{4\left(1-\frac{d^{2}-1}{d^{2}} p\right)}(\Delta H)^{2}+\frac{d(1-p)^{2}}{p} \operatorname{Tr}\left(H^{2}\right)
$$

where the second term increases linearly with respect to $d$, meaning the RLD QFI only provides a close bound for a small subsystem dimension.

\section{Example: Thermodynamic codes}

Finally, we provide an example that saturates the lower bound for single-error erasure noise channels in the small infidelity limit and matches the scaling of the lower bound for single-error depolarizing noise channels, while previously only the scaling optimality for erasure channels was demonstrated [10].

We consider the following two-dimensional thermodynamic code [10, 19, 68]

$$
\begin{gathered}
\mathcal{E}_{S \leftarrow L}\left(\left|0_{L}\right\rangle\right)=\left|g_{0}\right\rangle=\left|m_{n}\right\rangle, \\
\mathcal{E}_{S \leftarrow L}\left(\left|1_{L}\right\rangle\right)=\left|g_{1}\right\rangle=\left|(-m)_{n}\right\rangle,
\end{gathered}
$$

where

$$
\left|( \pm m)_{n}\right\rangle=\left(\begin{array}{c}
n \\
\frac{n \pm m}{2}
\end{array}\right)^{-\frac{1}{2}} \sum_{j: \sum_{k} j_{k}= \pm m}|\boldsymbol{j}\rangle,
$$

and $\boldsymbol{j}=\left(j_{1}, j_{2}, \ldots, j_{n}\right) \in\{-1,1\}^{n}$. The logical subspace is spanned by two Dicke states with different values of the total angular momentum along the z-axis. We also assume $n+m$ is an even number and $3 \leq m \ll N$. It is a covariant code whose physical and logical Hamiltonians are

$$
H_{S}=\sum_{k=1}^{n}\left(\sigma_{z}\right)_{S_{k}}, \quad H_{L}=m Z_{L}
$$

where $\sigma_{z}=|1\rangle\langle 1|-|-1\rangle\langle-1|$.

Let $\left|g_{0, \pm 1}^{(k)}\right\rangle=\left|(m \pm 1)_{n-1}\right\rangle_{S \backslash S_{k}}|\operatorname{vac}\rangle_{S_{k}},\left|g_{1, \pm 1}^{(k)}\right\rangle=\left|(-m \pm 1)_{n-1}\right\rangle_{S \backslash S_{k}}|\operatorname{vac}\rangle_{S_{k}}$, which represent the logical states after an erasure error occurs on $S_{k}$, and $\Pi^{\perp}$ be the projector onto the orthogonal subspace of $\operatorname{span}\left\{\left|g_{0, \pm 1}^{(k)}\right\rangle,\left|g_{1, \pm 1}^{(k)}\right\rangle, \forall k\right\}$. Consider the erasure noise channel $\mathcal{M}_{S}=\frac{1}{n} \sum_{k=1}^{n} \mathcal{M}_{S_{k}}$ where $\mathcal{M}_{S_{k}}\left(\rho_{S_{k}}\right)=|\operatorname{vac}\rangle\left\langle\left.\operatorname{vac}\right|_{S_{k}}\right.$ and the recovery channel

$$
\mathcal{R}_{L \leftarrow S}\left(\rho_{S}\right)=\sum_{k=1}^{n} \sum_{i, i^{\prime}=0}^{1} \sum_{j= \pm 1}\left|g_{i}\right\rangle\left\langle g_{i, j}^{(k)}\left|\rho_{S}\right| g_{i^{\prime}, j}^{(k)}\right\rangle\left\langle g_{i^{\prime}}\left|+\operatorname{Tr}\left(\Pi^{\perp} \rho_{S} \Pi^{\perp}\right)\right| g_{0}\right\rangle\left\langle g_{0}\right|,
$$

which maps the state $\left|g_{i, \pm 1}^{(k)}\right\rangle$ to $\left|g_{i}\right\rangle$ for all $k$. Then we could verify that $\mathcal{R}_{L \leftarrow S} \circ \mathcal{M}_{S} \circ \mathcal{E}_{S \leftarrow L}=$ $\mathcal{D}_{p, 0}$ with $p=\frac{1}{2}\left(1-\sqrt{1-\frac{m^{2}}{n^{2}}}\right)$. Using the relation between the noise rate $p$ and the worst-case entanglement fidelity of a dephasing channel (see Appx. B), we must have

$$
\begin{aligned}
\varepsilon\left(\mathcal{M}_{S}, \mathcal{E}_{S \leftarrow L}\right) & \leq 1-f^{2}\left(\mathcal{R}_{L \leftarrow S} \circ \mathcal{M}_{S} \circ \mathcal{E}_{S \leftarrow L}, \mathbb{1}_{L}\right) \\
& =\frac{1}{2}\left(1-\sqrt{1-\frac{m^{2}}{n^{2}}}\right)=\frac{m^{2}}{4 n^{2}}+O\left(\frac{m^{4}}{n^{4}}\right) .
\end{aligned}
$$

On the other hand, the lower bound (Eq. (63)) for $\varepsilon=\varepsilon\left(\mathcal{M}_{S}, \mathcal{E}_{S \leftarrow L}\right)$ is given by

$$
\varepsilon \geq \ell_{1}\left(\frac{m^{2}}{4 n^{2}}\right)=\frac{m^{2}}{4 n^{2}}+O\left(\frac{m^{4}}{n^{4}}\right)
$$


which is saturated asymptotically when $m / N \rightarrow 0$.

Next, we consider the single-error depolarizing noise channel $\mathcal{M}_{S}=\frac{1}{n} \sum_{k=1}^{n} \mathcal{M}_{S_{k}}$ where $\mathcal{M}_{S_{k}}\left(\rho_{S_{k}}\right)=\frac{\mathbb{1}}{2}$. It is in general difficult to write down the optimal recovery map explicitly. Instead, in order to calculate $\varepsilon\left(\mathcal{M}_{S}, \mathcal{E}_{S \leftarrow L}\right)$, we apply Corollary 2 in [20] to calculate an upper bound on the infidelity of thermodynamic codes in the limit $m / N \rightarrow 0$ and we obtain (see details in Appx. G)

$$
\varepsilon\left(\mathcal{M}_{S}, \mathcal{E}_{S \leftarrow L}\right) \leq \frac{3 m^{2}}{4 n^{2}}+o\left(\frac{m^{2}}{n^{2}}\right),
$$

which also matches the scaling of our lower bound for depolarizing noise channels (Eq. (68)), i.e., $\varepsilon\left(\mathcal{M}_{S}, \mathcal{E}_{S \leftarrow L}\right) \geq \frac{3 m^{2}}{8 n^{2}}+O\left(\frac{m^{4}}{n^{4}}\right)$.

\section{Conclusions and outlook}

In this paper, we advanced the understanding of covariant QEC by leveraging insights and techniques from quantum metrology and quantum resource theory. We first presented covariant QEC as a special type of metrological protocol where the sensitivity in parameter estimation could be linked to the code infidelity. We took inspirations from recent developments in quantum channel estimation: a no-go theorem [28-34] on the existence of perfect QEC was discovered based on a relation between Hamiltonians and noises (the HKS condition) which leads to a no-go theorem for covariant QEC; efficiently computable QFIs of quantum channels were also proposed [34, 36, 38], which leads to efficiently computable lower bounds for the code infidelity under generic noise channels. We also demonstrated how covariant QEC can be understood from an operational resource theory perspective, where the key insight is that there are fundamental limits on the distillation of pure coherent states using noisy ones $[42,44]$. The lower bounds we derived not only have a broad range of applications, but also improve upon previous lower bounds, which also lead to an improved approximate Eastin-Knill theorem that may be of particular interest in quantum computation.

In our metrological proof of the infidelity lower bounds, we reduced noisy quantum channels to rotated dephasing channels using one noiseless ancillary qubit. It in turn provided an entanglementassisted metrological protocol for channel estimation which might be of independent interest in quantum metrology. One implication of it is that known covariant codes might help improve the lower bounds for the channel QFIs, in situations where they are hard to calculate. Conversely, it indicates that lower bounds on the code infidelity might be improved if a separation between the entanglement-assisted QFIs with respect to one noiseless ancillary qubit and those with respect to an unbounded ancillary system could be identified.

There are still many open questions and future directions in the study of covariant QEC. First, it is not known, whether the HKS condition, which was shown to be sufficient for the non-existence of perfect covariant QEC codes, is also necessary. There are some examples of perfect covariant QEC codes, such as the [[4,2,2]] QEC code under single-qubit erasure noise [10,69], repetition codes under bit-flip noise $[48-50,52]$, but it is not yet clear how to generalize those examples. On the other hand, when the HKS condition is satisfied, it would also be desirable to obtain a systematic procedure to construct covariant codes saturating the infidelity lower bounds, at least in terms of scaling [12]. From the resource theory perspective, it would be interesting to investigate whether different monotones may induce other useful bounds, and whether invoking the resource theory of channels (see e.g., $[70,71]$ ) approaches may lead to new insights. It would also be important to further explore possible implications of the limitations on covariant QEC for physics, where symmetries naturally play prominent roles in a wide range of scenarios.

Note added. During the completion of this work, an independent work by Kubica and DemkowiczDobrzanski [47] appeared on arXiv, where a lower bound on the infidelity of covariant codes was also derived using tools from quantum metrology. Note that we employed different techniques and obtained lower bounds with a quadratic advantage in the small infidelity limit over the one in [47]. The bound in [47] was recently improved in [72] by quantifying the code inaccuracy using the diamond norm. Note that our bound still performs better in the small infidelity limit because $1-f^{2}(|\psi\rangle, \rho) \leq \frac{1}{2} \||\psi\rangle\langle\psi|-\rho \|_{1}$. 


\section{Acknowledgments}

We thank Victor V. Albert, Sepehr Nezami, John Preskill, Beni Yoshida, David Layden and Junyu Liu for helpful discussions. SZ and LJ acknowledge support from the ARL-CDQI (W911NF15-2-0067), ARO (W911NF-18-1-0020, W911NF-18-1-0212), ARO MURI (W911NF-16-1-0349), AFOSR MURI (FA9550-15-1-0015, FA9550-19-1-0399), DOE (DE-SC0019406), NSF (EFMA1640959, OMA-1936118), and the Packard Foundation (2013-39273). ZWL is supported by Perimeter Institute for Theoretical Physics. Research at Perimeter Institute is supported by the Government of Canada through Industry Canada and by the Province of Ontario through the Ministry of Research and Innovation.

\section{A Additivity of the regularized SLD QFI}

Here we prove the additivity of the regularized SLD QFI:

$$
F_{\mathscr{S}}^{\mathrm{reg}}\left(\mathcal{N}_{\theta} \otimes \tilde{\mathcal{N}}_{\theta}\right)=F_{\mathscr{S}}^{\mathrm{reg}}\left(\mathcal{N}_{\theta}\right)+F_{\mathscr{S}}^{\mathrm{reg}}\left(\tilde{\mathcal{N}}_{\theta}\right),
$$

for arbitrary quantum channels $\mathcal{N}_{\theta}$ and $\tilde{\mathcal{N}}_{\theta}$.

First, according to the additivity of the state QFI, we must have

$$
F_{\mathscr{S}}^{\mathrm{reg}}\left(\mathcal{N}_{\theta} \otimes \tilde{\mathcal{N}}_{\theta}\right) \geq F_{\mathscr{S}}^{\mathrm{reg}}\left(\mathcal{N}_{\theta}\right)+F_{\mathscr{S}}^{\mathrm{reg}}\left(\tilde{\mathcal{N}}_{\theta}\right) \text {. }
$$

Thus, we only need to prove

$$
F_{\mathscr{S}}^{\mathrm{reg}}\left(\mathcal{N}_{\theta} \otimes \tilde{\mathcal{N}}_{\theta}\right) \leq F_{\mathscr{S}}^{\mathrm{reg}}\left(\mathcal{N}_{\theta}\right)+F_{\mathscr{S}}^{\mathrm{reg}}\left(\tilde{\mathcal{N}}_{\theta}\right) .
$$

We use the following definition of the regularized SLD QFI [29, 30, 34] (which is equivalent to Eq. (12))

$$
F_{\mathscr{S}}^{\mathrm{reg}}\left(\mathcal{N}_{\theta}\right)= \begin{cases}4 \min _{\mathbf{K}^{\prime}: \beta=0}\|\alpha\|, & i \sum_{i=1}^{r}\left(\partial_{\theta} K_{i}\right)^{\dagger} K_{i} \in \operatorname{span}\left\{K_{i}^{\dagger} K_{j}, \forall i, j\right\} \\ +\infty & \text { otherwise }\end{cases}
$$

where $\mathbf{K}^{\prime}$ is any set of Kraus operators representing $\mathcal{N}_{\theta}, \alpha=\sum_{i=1}^{r}\left(\partial_{\theta} K_{i}^{\prime}\right)^{\dagger}\left(\partial_{\theta} K_{i}^{\prime}\right)$ and $\beta=$ $i \sum_{i=1}^{r}\left(\partial_{\theta} K_{i}^{\prime}\right)^{\dagger} K_{i}^{\prime}$. Without loss of generality, assume both $F_{\mathscr{S}}^{\mathrm{reg}}\left(\mathcal{N}_{\theta}\right)$ and $F_{\mathscr{S}}^{\mathrm{reg}}\left(\tilde{\mathcal{N}}_{\theta}\right)$ are finite, i.e., $i \sum_{i=1}^{r}\left(\partial_{\theta} K_{i}\right)^{\dagger} K_{i} \in \operatorname{span}\left\{K_{i}^{\dagger} K_{j}, \forall i, j\right\}$ and $i \sum_{i=1}^{\tilde{r}}\left(\partial_{\theta} \tilde{K}_{i}\right)^{\dagger} \tilde{K}_{i} \in \operatorname{span}\left\{\tilde{K}_{i}^{\dagger} \tilde{K}_{j}, \forall i, j\right\}$. We first note that $F_{\mathscr{S}}^{\mathrm{reg}}\left(\mathcal{N}_{\theta} \otimes \tilde{\mathcal{N}}_{\theta}\right)$ is also finite, because

$$
\begin{aligned}
i \sum_{i=1}^{r} \sum_{j=1}^{\tilde{r}}\left(\partial_{\theta}\left(K_{i} \otimes \tilde{K}_{j}\right)\right)^{\dagger}\left(K_{i} \otimes \tilde{K}_{j}\right) & =i \sum_{i=1}^{r}\left(\partial_{\theta} K_{i}\right)^{\dagger} K_{i} \otimes \mathbb{1}+i \sum_{j=1}^{\tilde{r}} \mathbb{1} \otimes\left(\partial_{\theta} \tilde{K}_{j}\right)^{\dagger} \tilde{K}_{j} \\
& \in \operatorname{span}\left\{\mathbb{1} \otimes K_{i}^{\dagger} K_{j}, \tilde{K}_{i}^{\dagger} \tilde{K}_{j} \otimes \mathbb{1}, \forall i, j\right\} .
\end{aligned}
$$

According to Eq. (84), there exists $\mathbf{K}^{\prime}$ and $\tilde{\mathbf{K}}^{\prime}$ such that $\beta=\tilde{\beta}=0$ and

$$
F_{\mathscr{S}}^{\mathrm{reg}}\left(\mathcal{N}_{\theta}\right)=4\|\alpha\|, \quad F_{\mathscr{S}}^{\mathrm{reg}}\left(\tilde{\mathcal{N}}_{\theta}\right)=4\|\tilde{\alpha}\| .
$$

Then $\tilde{\tilde{K}}_{i j}^{\prime}=K_{i}^{\prime} \otimes \tilde{K}_{j}^{\prime}$ is a set of Kraus operators representing $\mathcal{N}_{\theta} \otimes \tilde{\mathcal{N}}_{\theta}$.

$$
\begin{gathered}
\tilde{\tilde{\alpha}}=\sum_{i=1}^{r} \sum_{j=1}^{\tilde{r}} \partial_{\theta}\left(\tilde{\tilde{K}}_{i j}\right)^{\dagger} \partial_{\theta}\left(\tilde{\tilde{K}}_{i j}\right)=\alpha \otimes \mathbb{1}+\mathbb{1} \otimes \tilde{\alpha}+2 \beta \otimes \tilde{\beta}=\alpha \otimes \mathbb{1}+\mathbb{1} \otimes \tilde{\alpha}, \\
\tilde{\tilde{\beta}}=\beta \otimes \mathbb{1}+\mathbb{1} \otimes \tilde{\beta}=0 .
\end{gathered}
$$

Therefore $F_{\mathscr{S}}^{\mathrm{reg}}\left(\mathcal{N}_{\theta} \otimes \tilde{\mathcal{N}}_{\theta}\right) \leq 4\|\tilde{\tilde{\alpha}}\|=4\|\alpha\|+4\|\tilde{\alpha}\|=F_{\mathscr{S}}^{\mathrm{reg}}\left(\mathcal{N}_{\theta}\right)+F_{\mathscr{S}}^{\mathrm{reg}}\left(\tilde{\mathcal{N}}_{\theta}\right)$. 


\section{B Worst-case entanglement fidelity for rotated dephasing channels}

Here we calculate the worst-case entanglement fidelity for rotated dephasing channels (Eq. (17))

$$
\mathcal{D}_{p, \phi}(\rho)=(1-p) e^{-i \frac{\phi}{2} Z} \rho e^{i \frac{\phi}{2} Z}+p e^{-i \frac{\phi}{2} Z} Z \rho Z e^{i \frac{\phi}{2} Z} .
$$

We use the following formula for the worst-case entanglement fidelity [45]:

$$
f^{2}\left(\mathcal{D}_{p, \phi}, \mathbb{1}\right)=\min _{|\psi\rangle}\left\langle\psi\left|\left(\mathcal{D}_{p, \phi} \otimes \mathbb{1}\right)(|\psi\rangle\langle\psi|)\right| \psi\right\rangle
$$

Let $|\psi\rangle=\alpha_{00}|00\rangle+\alpha_{01}|01\rangle+\alpha_{10}|10\rangle+\alpha_{11}|11\rangle$, then

$$
\begin{aligned}
& \left(\mathcal{D}_{p, \phi} \otimes \mathbb{1}\right)(|\psi\rangle\langle\psi|)= \\
& \quad\left(\begin{array}{cccc}
\alpha_{00} \alpha_{00}^{*} & \alpha_{00} \alpha_{01}^{*} & (1-2 p) e^{-i \phi} \alpha_{00} \alpha_{10}^{*} & (1-2 p) e^{-i \phi} \alpha_{00} \alpha_{11}^{*} \\
\alpha_{00} \alpha_{01}^{*} & \alpha_{01} \alpha_{01}^{*} & (1-2 p) e^{-i \phi} \alpha_{01} \alpha_{10}^{*} & (1-2 p) e^{-i \phi} \alpha_{01} \alpha_{11}^{*} \\
(1-2 p) e^{i \phi} \alpha_{10} \alpha_{00}^{*} & (1-2 p) e^{i \phi} \alpha_{10} \alpha_{01}^{*} & \alpha_{10} \alpha_{10}^{*} & \alpha_{10} \alpha_{11}^{*} \\
(1-2 p) e^{i \phi} \alpha_{11} \alpha_{00}^{*} & (1-2 p) e^{i \phi} \alpha_{11} \alpha_{01}^{*} & \alpha_{11} \alpha_{10}^{*} & \alpha_{11} \alpha_{11}^{*}
\end{array}\right) .
\end{aligned}
$$

Then

$$
\begin{aligned}
1-f^{2}\left(\mathcal{D}_{p, \phi}, \mathbb{1}\right) & =\max _{\alpha_{00,01,10,11}} 2 \operatorname{Re}\left[\left(1-(1-2 p) e^{-i \phi}\right)\right]\left(\left|\alpha_{00}\right|^{2}+\left|\alpha_{01}\right|^{2}\right)\left(\left|\alpha_{10}\right|^{2}+\left|\alpha_{11}\right|^{2}\right) \\
& =\frac{1}{2}(1-(1-2 p) \cos \phi) \geq p
\end{aligned}
$$

\section{An upper bound on the regularized SLD QFI for multi-error noise channels}

Consider the following types of noise channels and physical Hamiltonians:

$$
\mathcal{M}_{S}=\sum_{\chi \in \mathscr{X}} q_{\chi} \mathcal{M}_{\chi}, \quad H_{S}=\sum_{\chi \in \mathscr{X}} H_{\chi} .
$$

where $q_{\chi}>0, \sum_{\chi \in \mathscr{X}}^{n} q_{\chi}=1, \mathscr{X}$ is a collection of sets of subsystems and $\mathcal{M}_{\chi}$ and $H_{\chi}$ act on the corresponding sets of subsystems. For example, in the single-error case, $\mathscr{X}$ is the collection of all local subsystems $\left\{\left\{S_{1}\right\},\left\{S_{2}\right\}, \ldots,\left\{S_{n}\right\}\right\}$.

Assume that the HKS condition is satisfied for each $\chi$, i.e., $H_{\chi} \in \operatorname{span}\left\{K_{\chi, i}^{\dagger} K_{\chi, j}, \forall i, j\right\}$ and that the set of Hamiltonians $\left\{H_{\chi}\right\}_{\chi \in \mathscr{X}}$ commute pairwise, i.e., $\left[H_{\chi}, H_{\chi^{\prime}}\right]=0$ for all $\chi, \chi^{\prime} \in \mathscr{X}$. We then derive an upper bound of $F_{\mathscr{S}}^{\mathrm{reg}}\left(\mathcal{M}_{S}, H_{S}\right)=4 \min _{h: \beta_{S}=0}\left\|\alpha_{S}\right\|$, where

$$
\alpha_{S}=\mathbf{K}_{S}^{\dagger} h^{2} \mathbf{K}_{S}-H_{S}^{2}, \text { and } \beta_{S}=\mathbf{K}_{S}^{\dagger} h \mathbf{K}_{S}-H_{S} .
$$

When the HKS condition is satisfied for each $\chi$, to derive an upper bound on $F_{\mathscr{S}}^{\mathrm{reg}}\left(\mathcal{M}_{S}, H_{S}\right)$, we can restrict $h$ to be a block diagonal matrix when partitioning the indices of Kraus operators according to $\mathscr{X}$. Then

$$
\alpha_{S}=\sum_{\chi} q_{\chi} \mathbf{K}_{\chi}^{\dagger} h_{\chi}^{2} \mathbf{K}_{\chi}-\left(\sum_{\chi} H_{\chi}\right)^{2}, \text { and } \beta_{S}=\sum_{\chi} q_{\chi} \mathbf{K}_{\chi}^{\dagger} h_{\chi} \mathbf{K}_{\chi}-H_{\chi} .
$$

Let $\beta_{\chi}=q_{\chi} \mathbf{K}_{\chi}^{\dagger} h_{\chi} \mathbf{K}_{\chi}-H_{\chi}$. Then

$$
\begin{aligned}
F_{\mathscr{S}}^{\mathrm{reg}}\left(\mathcal{M}_{S}, H_{S}\right) & =4 \min _{h: \beta_{S}=0}\left\|\alpha_{S}\right\| \leq 4 \min _{h_{\chi}: \beta_{\chi}=0}\left\|\alpha_{S}\right\| \\
& \leq 4 \min _{h_{\chi}: \beta_{\chi}=0}\left\|\sum_{\chi} q_{\chi} \mathbf{K}_{\chi}^{\dagger} h_{\chi}^{2} \mathbf{K}_{\chi}\right\| \leq 4 \sum_{\chi \in \mathscr{X}} \min _{h_{\chi}: \beta_{\chi}=0}\left\|q_{\chi} \mathbf{K}_{\chi}^{\dagger} h_{\chi}^{2} \mathbf{K}_{\chi}\right\| .
\end{aligned}
$$

The upper bound on the right-hand side of Eq. (97) is efficiently computable when the size of each set $\chi$ is small. However, given $\mathscr{X}$ and $H_{S}$, it is in general not clear how to decompose $H_{S}$ 
into $\sum_{\chi \in \mathscr{X}} H_{\chi}$ in order to attain the optimal upper bound, and the upper bound might not be tight.

Now we provide an example, where we compute the upper bound of the regularized SLD QFI for $t$ erasure errors and identical local Hamiltonians $H$. We have

$$
\mathcal{M}_{S}=\sum_{\chi \in \mathscr{X}} q_{\chi} \mathcal{M}_{\chi}, \quad H_{S}=\sum_{k=1}^{n} H_{S_{k}}=\sum_{\chi \in \mathscr{X}} H_{\chi} .
$$

where $\mathscr{X}$ is the collection of all size- $t$ sets of subsystems, $\mathcal{M}_{\chi}$ is the completely erasure channel on the corresponding set, $q_{\chi}=1 /\left(\begin{array}{c}n \\ t\end{array}\right)$ for all $\chi, H_{\chi}=\sum_{i=1}^{t} H_{\chi_{i}} /\left(\begin{array}{c}n-1 \\ t-1\end{array}\right)$ and $H_{S_{k}}$ is identical for each subsystem which we denote by $H$. Suppose $\beta_{\chi}=q_{\chi} \mathbf{K}_{\chi}^{\dagger} h_{\chi} \mathbf{K}_{\chi}-H_{\chi}$, where $K_{\chi,\left(i_{1} \cdots i_{t}\right)}=$ $\left(\left|\operatorname{vac}^{\otimes t}\right\rangle_{\chi}\left\langle\left. i_{1} \cdots i_{t}\right|_{\chi}\right)\right.$. Then $h_{\chi}=H_{\chi} / q_{\chi}$ and $q_{\chi} \mathbf{K}_{\chi}^{\dagger} h_{\chi}^{2} \mathbf{K}_{\chi}=H_{\chi}^{2} / q_{\chi}$. We have

$$
\begin{aligned}
F_{\mathscr{S}}^{\mathrm{reg}}\left(\mathcal{M}_{S}, H_{S}\right) & \leq 4 \sum_{\chi \in \mathscr{X}} \min _{h_{\chi}: \beta_{\chi}=0}\left\|q_{\chi} \mathbf{K}_{\chi}^{\dagger} h_{\chi}^{2} \mathbf{K}_{\chi}\right\| \\
& =4\|H\|^{2}\left(\begin{array}{c}
n \\
t
\end{array}\right)^{2} \frac{t^{2}}{\left(\begin{array}{c}
n-1 \\
t-1
\end{array}\right)^{2}}=4\|H\|^{2} n^{2}=(\Delta H)^{2} n^{2},
\end{aligned}
$$

It implies that

$$
\varepsilon \geq \ell_{1}\left(\frac{\left(\Delta H_{L}\right)^{2}}{4 n^{2}(\Delta H)^{2}}\right)
$$

for arbitrary $t$, which matches our bound (Eq. (63)) in the $t=1$ case. It is unclear though, whether the bound is tight. Note that a similar bound for $t$ erasure errors were derived in [72].

\section{Regularized SLD QFI for erasure and single-qubit depolarizing chan- nels}

Here we calculate the SLD QFI for erasure and depolarizing channels. We first calculate $F_{\mathscr{S}}^{\mathrm{reg}}\left(\mathcal{N}^{\mathrm{e}}, H\right)$ where $\mathcal{N}^{\mathrm{e}}=(1-p) \rho+p|\operatorname{vac}\rangle\langle\operatorname{vac}|$. Using the Kraus operators in Eq. (59),

$$
\beta=\mathbf{K}^{\dagger} h \mathbf{K}-H \quad \Leftrightarrow \quad h=\left(\begin{array}{cc}
\frac{h_{11}}{1-p} & 0 \\
0 & \frac{H-h_{11} \mathbb{1}}{p}
\end{array}\right) .
$$

Then

$$
\begin{gathered}
\alpha=\mathbf{K}^{\dagger} h^{2} \mathbf{K}-H^{2}=\frac{h_{11}^{2}}{1-p}+\frac{\left(H-h_{11} \mathbb{1}\right)^{2}}{p}-H^{2}=\frac{1-p}{p} H^{2}-\frac{2 h_{11}}{p} H+\frac{h_{11}^{2}}{p(1-p)}, \\
F_{\mathscr{S}}^{\mathrm{reg}}\left(\mathcal{N}^{\mathrm{e}}, H\right)=4 \min _{h_{11}}\|\alpha\|=4 \max _{\rho} \min _{h_{11}} \operatorname{Tr}(\rho \alpha) \\
=4 \max _{\rho} \frac{1-p}{p}\left(\operatorname{Tr}\left(H^{2} \rho\right)-\operatorname{Tr}(\rho H)^{2}\right)=\frac{1-p}{p}(\Delta H)^{2},
\end{gathered}
$$

where we use the minimax theorem $[73,74]$ in the second step.

We use the formula in $\operatorname{Sec} . \operatorname{VII}(\mathrm{A})$ in [34] to calculate the regularized SLD QFI $F_{\mathscr{S}}^{\mathrm{reg}}\left(\mathcal{N}^{\mathrm{d}}, H\right)$ for single-qubit depolarizing channels $\mathcal{N}^{\mathrm{d}}(\rho)=(1-p) \rho+p \frac{\mathbb{1}}{2}$.

$$
F_{\mathscr{S}}^{\mathrm{reg}}\left(\mathcal{N}^{\mathrm{d}}, H\right)=(\Delta H)^{2} \frac{1-w}{w}
$$

where $w=4\left(\frac{y^{2}}{2 y}+\frac{x y}{x+y}\right)$ with $x=1-\frac{3}{4} p$ and $y=\frac{p}{4}$. Then $F_{\mathscr{S}}^{\mathrm{reg}}\left(\mathcal{N}^{\mathrm{d}}, H\right)=(\Delta H)^{2} \frac{2(1-p)^{2}}{p(3-2 p)}$. 


\section{E Regularized SLD QFI for general depolarizing channels}

Here we prove an upper bound on $F_{\mathscr{S}}^{\text {reg }}\left(\mathcal{N}^{\mathrm{d}}, H\right)$ for general depolarizing channels $\mathcal{N}^{\mathrm{d}}(\rho)=(1-$ p) $\rho+p \frac{\mathbb{1}}{d}$ with the Kraus operators

$$
K_{1}=\sqrt{x} \mathbb{1}, \quad K_{i}=\sqrt{y} U_{i-1}, \forall 2 \leq i \leq d^{2},
$$

where we define $x=1-\frac{d^{2}-1}{d^{2}} p, y=\frac{1}{d^{2}} p$.

Any $\tilde{h}$ satisfying $\tilde{\beta}=\mathbf{K}^{\dagger} \tilde{h} \mathbf{K}-H=0$ provides an upper bound on $F_{\mathscr{S}}^{\mathrm{reg}}\left(\mathcal{N}^{\mathrm{d}}, H\right)$ through

$$
F_{\mathscr{S}}^{\mathrm{reg}}\left(\mathcal{N}^{\mathrm{d}}, H\right)=4 \min _{h: \beta=0}\|\alpha\| \leq\left. 4\|\alpha\|\right|_{h=\tilde{h}} .
$$

To find a suitable $\tilde{h}$ which provides a good upper bound on $F_{\mathscr{S}}^{\mathrm{reg}}\left(\mathcal{N}^{\mathrm{d}}, H\right)$, we use $\tilde{h}$ which is the solution of

$$
4 \min _{h: \beta=0} \operatorname{Tr}(\alpha)
$$

The solution of Eq. (107) is

$$
\tilde{h}=\frac{1}{2 z d}\left(\begin{array}{cccc}
0 & \frac{\sqrt{x y}}{x+y} \operatorname{Tr}\left(H U_{1}^{\dagger} U_{0}\right) & \cdots & \frac{\sqrt{x y}}{x+y} \operatorname{Tr}\left(H U_{d^{2}-1}^{\dagger} U_{0}\right) \\
\frac{\sqrt{x y}}{x+y} \operatorname{Tr}\left(H U_{0}^{\dagger} U_{1}\right) & 0 & \cdots & \frac{1}{2} \operatorname{Tr}\left(H U_{d^{2}-1}^{\dagger} U_{1}\right) \\
\vdots & \vdots & \ddots & \vdots \\
\frac{\sqrt{x y}}{x+y} \operatorname{Tr}\left(H U_{0}^{\dagger} U_{d^{2}-1}\right) & \frac{1}{2} \operatorname{Tr}\left(H U_{1}^{\dagger} U_{d^{2}-1}\right) & \cdots & 0
\end{array}\right) \text {, }
$$

where $z=\frac{x y}{x+y}+\frac{y\left(d^{2}-2\right)}{4}$ and we used the assumption $\operatorname{Tr}(H)=0$ and

$$
\mathbf{K}^{\dagger} \tilde{h}^{2} \mathbf{K}=\left(\frac{1}{4 z}-\frac{y}{4 z^{2}}\left(\frac{1}{4}-\frac{x y}{(x+y)^{2}}\right)-1\right) H^{2}+\frac{y}{4 z^{2} d}\left(\frac{x}{x+y}-\frac{1}{2}\right)^{2} \operatorname{Tr}\left(H^{2}\right) \mathbb{1} .
$$

Using $\left\|H^{2}\right\|=\frac{(\Delta H)^{2}}{4}$ and $\operatorname{Tr}\left(H^{2}\right) \leq \frac{d}{4}(\Delta H)^{2}$,

$$
F_{\mathscr{S}}^{\mathrm{reg}}\left(\mathcal{N}^{\mathrm{d}}, H\right) \leq 4\|\alpha\| \leq(\Delta H)^{2}\left(\frac{1}{4 z}-1\right)=(\Delta H)^{2} \frac{d^{2}(1-p)^{2}}{p\left(d^{2}(1-p)+2\right)} \leq(\Delta H)^{2}\left(\frac{1-p}{p}\right),
$$

upper bounded by the $F_{\mathscr{S}}^{\text {reg }}\left(\mathcal{N}^{\mathrm{d}}, H\right)$ for erasure channels (Eq. (60)).

\section{F Improved approximate Eastin-Knill theorem}

Here we derive specific lower bounds on the infidelity of codes covariant with respect to unitary groups which lead to new approximate Eastin-Knill theorems, following the discussion in [10].

$S U\left(d_{L}\right)$-covariant codes in an $n$-partite system $S$ are defined by the encoding channels $\mathcal{E}_{S \leftarrow L}$ which satisfy

$$
\mathcal{E}_{S \leftarrow L}\left(U_{L}(g)(\cdot) U_{L}^{\dagger}(g)\right)=\left(\bigotimes_{k=1}^{n} U_{S_{k}}(g)\right) \mathcal{E}_{S \leftarrow L}(\cdot)\left(\bigotimes_{k=1}^{n} U_{S_{k}}^{\dagger}(g)\right), \forall g \in S U\left(d_{L}\right),
$$

where $U_{S_{k}}(g)$ and $U_{L}(g)$ are unitary representations of $S U\left(d_{L}\right)$. It was shown in Theorem 18 in the Supplemental Material of [10] that fixing $H_{L}=\operatorname{diag}(1,0, \ldots,-1)$ and letting $H_{S_{k}}$ be the corresponding generator acting on the subsystem $k$, we have

$$
d_{k} \geq\left(\begin{array}{c}
d_{L}-1+\left\lceil\left\|H_{S_{k}}\right\|\right\rceil \\
d_{L}-1
\end{array}\right)
$$

where $\left\lceil\left\|H_{S_{k}}\right\|\right\rceil$ denotes the closest integer no smaller than $\left\|H_{S_{k}}\right\|$. Using the inequality $\left(\begin{array}{c}a+b \\ a\end{array}\right) \geq$ $\left(1+\frac{b}{a}\right)^{a}$,

$$
\begin{gathered}
d_{k} \geq\left(\frac{d_{L}-1+\left\lceil\left\|H_{S_{k}}\right\|\right\rceil}{d_{L}-1}\right)^{d_{L}-1}, \Rightarrow \quad\left(\exp \left(\frac{\ln d_{k}}{d_{L}-1}\right)-1\right)\left(d_{L}-1\right) \geq\left\|H_{S_{k}}\right\|, \\
\Rightarrow \sum_{k=1}^{n}\left(\exp \left(\frac{\ln d_{k}}{d_{L}-1}\right)-1\right)^{2}\left(d_{L}-1\right)^{2} \geq \frac{1}{4} \sum_{k}\left(\Delta H_{S_{k}}\right)^{2} .
\end{gathered}
$$


Then using Eq. (63), we have for any $\varepsilon \geq \varepsilon\left(\mathcal{M}_{S}, \mathcal{E}_{S \leftarrow L}\right)$,

$$
\varepsilon \geq \ell_{1}\left(\frac{1}{4 n \sum_{k=1}^{n}\left(\exp \left(\frac{\ln d_{k}}{d_{L}-1}\right)-1\right)^{2}\left(d_{L}-1\right)^{2}}\right) .
$$

For large $d_{L}=\Omega\left(\ln d_{k}\right)$,

$$
\begin{aligned}
\varepsilon & \geq \ell_{1}\left(\frac{1}{4 n \sum_{k=1}^{n}\left(\ln d_{k}\right)^{2}}+O\left(\frac{1}{n d_{L} \sum_{k=1}^{n} \ln d_{k}}\right)\right) \\
& =\frac{1}{4 n \sum_{k=1}^{n}\left(\ln d_{k}\right)^{2}}+O\left(\frac{1}{n d_{L} \sum_{k=1}^{n} \ln d_{k}}+\frac{1}{n^{2}\left(\sum_{k=1}^{n}\left(\ln d_{k}\right)^{2}\right)^{2}}\right) .
\end{aligned}
$$

Compared to Theorem 4 in [10]:

$$
\varepsilon \geq\left(\frac{1}{2 n \max _{k} \ln d_{k}}+O\left(\frac{1}{n d_{L}}\right)\right)^{2}=\frac{1}{4 n^{2}\left(\max _{k} \ln d_{k}\right)^{2}}+O\left(\frac{1}{n^{2} d_{L} \max _{k} \ln d_{k}}\right)
$$

our bound improves the maximum of $\ln d_{k}$ in the denominator to their quadratic mean. Moreover, it works for not only single-error erasure noise channel $\mathcal{M}_{S}=\sum_{k=1}^{n} \frac{1}{n} \mathcal{M}_{S_{k}}$ where $\mathcal{M}_{S_{k}}(\cdot)=$ $|\operatorname{vac}\rangle\left\langle\left.\operatorname{vac}\right|_{S_{k}}\right.$, but also single-error depolarizing noise channel $\mathcal{M}_{S}=\sum_{k=1}^{k} \frac{1}{n} \mathcal{M}_{S_{k}}$ where $\mathcal{M}_{S_{k}}(\cdot)=$ $\frac{\mathbb{1}}{d_{k}}$.

\section{G Infidelity of thermodynamic codes under depolarizing noise}

Here we use Corollary 2 from [20] to calculate the infidelity of thermodynamic codes under depolarizing noise channels in the limit $m / N \rightarrow 0$ :

Lemma 4 ([20]). A code defined by its projector $P$ is $\varepsilon$-correctable under a noise channel $\mathcal{M}(\cdot)=$ $\sum_{i=1}^{r} K_{i}(\cdot) K_{i}^{\dagger}$ if and only if $P K_{i}^{\dagger} K_{j} P=A_{i j} P+P \delta A_{i j} P$ for some $A_{i j}$ and $\delta A_{i j}$ where $A_{i j}$ are the components of a density operator, and $1-f^{2}(\mathcal{A}+\delta \mathcal{A}, \mathcal{A}) \leq \varepsilon$ where $\mathcal{A}(\rho)=\sum_{i j} A_{i j} \operatorname{Tr}(\rho)|i\rangle\langle j|$ and $(\mathcal{A}+\delta \mathcal{A})(\rho)=\mathcal{A}(\rho)+\sum_{i j} \operatorname{Tr}\left(\rho \delta A_{i j}\right)|i\rangle\langle j|$.

Let $P=\left|g_{0}\right\rangle\left\langle g_{0}|+| g_{1}\right\rangle\left\langle g_{1}\right|, \mathcal{M}=\mathcal{M}_{S}$ with Kraus operators

$$
K_{k, i}=\frac{1}{2 \sqrt{n}}\left(U_{i}\right)_{S_{k}}, \quad i=0,1,2,3
$$

where $U_{0}, U_{1}, U_{2}, U_{3}$ are respectively $\mathbb{1}, \sigma_{x}=|1\rangle\langle-1|+|-1\rangle\left\langle 1\left|, \sigma_{y}=-i\right| 1\right\rangle\langle-1|+i|-1\rangle\langle 1|$, and $\sigma_{z}=|1\rangle\langle 1|-|-1\rangle\langle-1|$.

For $m \geq 3,\left\langle g_{0}|E| g_{1}\right\rangle=0$ for any operator $E$ acting on at most two qubits. Here we consider $\delta A_{i j} \propto\left(\left|g_{0}\right\rangle\left\langle g_{0}|-| g_{1}\right\rangle\left\langle g_{1}\right|\right)$. That is, let $\delta A_{i j}=B_{i j}\left(\left|g_{0}\right\rangle\left\langle g_{0}|-| g_{1}\right\rangle\left\langle g_{1}\right|\right)$. $A$ and $B$ are $4 n \times 4 n$ matrices

$$
A=\left(\begin{array}{llll}
A^{(0,0)} & A^{(0,1)} & A^{(0,2)} & A^{(0,3)} \\
A^{(1,0)} & A^{(1,1)} & A^{(1,2)} & A^{(1,3)} \\
A^{(2,0)} & A^{(2,1)} & A^{(2,2)} & A^{(2,3)} \\
A^{(3,0)} & A^{(3,1)} & A^{(3,2)} & A^{(3,3)}
\end{array}\right), \quad B=\left(\begin{array}{llll}
B^{(0,0)} & B^{(0,1)} & B^{(0,2)} & B^{(0,3)} \\
B^{(1,0)} & B^{(1,1)} & B^{(1,2)} & B^{(1,3)} \\
B^{(2,0)} & B^{(2,1)} & B^{(2,2)} & B^{(2,3)} \\
B^{(3,0)} & B^{(3,1)} & B^{(3,2)} & B^{(3,3)}
\end{array}\right)
$$

where

$$
\begin{aligned}
A_{k k^{\prime}}^{(i, j)} & =\frac{1}{2}\left(\left\langle g_{0}\left|K_{k, i}^{\dagger} K_{k^{\prime}, j}\right| g_{0}\right\rangle+\left\langle g_{1}\left|K_{k, i}^{\dagger} K_{k^{\prime}, j}\right| g_{1}\right\rangle\right), \\
B_{k k^{\prime}}^{(i, j)} & =\frac{1}{2}\left(\left\langle g_{0}\left|K_{k, i}^{\dagger} K_{k^{\prime}, j}\right| g_{0}\right\rangle-\left\langle g_{1}\left|K_{k, i}^{\dagger} K_{k^{\prime}, j}\right| g_{1}\right\rangle\right),
\end{aligned}
$$

so that $P K_{i}^{\dagger} K_{j} P=A_{i j} P+P \delta A_{i j} P$ holds. 
A detailed calculation shows that $A^{(i, j)}=0$ when $i \neq j, B^{(i, j)}=0$ when $i+j \neq 3$, and

$$
\begin{aligned}
& A^{(0,0)}=\frac{1}{4 n}\left(\begin{array}{cccc}
1 & 1 & \cdots & 1 \\
1 & 1 & \cdots & 1 \\
\vdots & \vdots & \ddots & \vdots \\
1 & 1 & \cdots & 1
\end{array}\right) \\
& A^{(1,1)}=A^{(2,2)}=\frac{1}{4 n}\left(\begin{array}{cccc}
1 & \frac{n^{2}-m^{2}}{2 n(n-1)} & \cdots & \frac{n^{2}-m^{2}}{2 n(n-1)} \\
\frac{n^{2}-m^{2}}{2 n(n-1)} & 1 & \cdots & \frac{n^{2}-m^{2}}{2 n(n-1)} \\
\vdots & \vdots & \ddots & \vdots \\
\frac{n^{2}-m^{2}}{2 n(n-1)} & \frac{n^{2}-m^{2}}{2 n(n-1)} & \cdots & 1
\end{array}\right) \text {, } \\
& A^{(3,3)}=\frac{1}{4 n}\left(\begin{array}{cccc}
1 & \frac{m^{2}-n}{n(n-1)} & \cdots & \frac{m^{2}-n}{n(n-1)} \\
\frac{m^{2}-n}{n(n-1)} & 1 & \cdots & \frac{m^{2}-n}{n(n-1)} \\
\vdots & \vdots & \ddots & \vdots \\
\frac{m^{2}-n}{n(n-1)} & \frac{m^{2}-n}{n(n-1)} & \cdots & 1
\end{array}\right) \\
& B^{(0,3)}=B^{(3,0)}=\frac{m}{4 n^{2}}\left(\begin{array}{cccc}
1 & 1 & \cdots & 1 \\
1 & 1 & \cdots & 1 \\
\vdots & \vdots & \ddots & \vdots \\
1 & 1 & \cdots & 1
\end{array}\right), \quad B^{(1,2)}=-B^{(2,1)}=i \frac{m}{4 n^{2}} \mathbb{1} \text {. }
\end{aligned}
$$

Next we note that

$$
\begin{aligned}
f(\mathcal{A}, \mathcal{A}+\delta \mathcal{A}) & =\min _{|\psi\rangle} f\left(\left(\mathcal{A} \otimes \mathbb{1}_{R}\right)(|\psi\rangle\langle\psi|),\left((\mathcal{A}+\delta \mathcal{A}) \otimes \mathbb{1}_{R}\right)(|\psi\rangle\langle\psi|)\right) \\
& =\min _{p_{i}, \rho_{i}, i=0,1} f\left(A \otimes\left(p_{0} \rho_{0}+p_{1} \rho_{1}\right), p_{0}(A+B) \otimes \rho_{0}+p_{1}(A-B) \otimes \rho_{1}\right) \\
& \geq \min _{p_{i}, \rho_{i}, i=0,1} p_{0} f(A, A+B)+p_{1} f(A, A-B)=f(A, A+B),
\end{aligned}
$$

where in the second step we define $\left\langle g_{i} \mid \psi\right\rangle\left\langle\psi \mid g_{i}\right\rangle=p_{i} \rho_{i}$ for $i=0,1$, and in the third step we use the joint concavity of fidelity and in the last step we use $f(A+B)=f(A-B)$. Therefore we must have

$$
f(\mathcal{A}, \mathcal{A}+\delta \mathcal{A})=f(A, A+B),
$$

by noticing that $f\left(\mathcal{A}\left(\left|g_{0}\right\rangle\left\langle g_{0}\right|\right),(\mathcal{A}+\delta \mathcal{A})\left(\left|g_{0}\right\rangle\left\langle g_{0}\right|\right)\right)=f(A, A+B)$. First note that $A^{(i, i)}$ and $B^{(i, j)}$ could be diagonalized in the following way:

$$
\begin{gathered}
A^{(0,0)}=\frac{1}{4 n}\left(n\left|\psi_{1}\right\rangle\left\langle\psi_{1}\right|\right), \quad B^{(0,3)}=B^{(3,0)}=\frac{m}{4 n}\left|\psi_{1}\right\rangle\left\langle\psi_{1}\right|, \\
A^{(1,1)}=A^{(2,2)}=\frac{1}{4 n}\left(\frac{n^{2}+2 n-m^{2}}{2 n}\left|\psi_{1}\right\rangle\left\langle\psi_{1}\left|+\frac{n^{2}-2 n+m^{2}}{2 n(n-1)} \sum_{k=2}^{n}\right| \psi_{k}\right\rangle\left\langle\psi_{k}\right|\right), \\
A^{(3,3)}=\frac{1}{4 n}\left(\frac{m^{2}}{n}\left|\psi_{1}\right\rangle\left\langle\psi_{1}\left|+\frac{n^{2}-m^{2}}{n(n-1)} \sum_{k=2}^{n}\right| \psi_{k}\right\rangle\left\langle\psi_{k}\right|\right),
\end{gathered}
$$

where $\left|\psi_{1}\right\rangle=\frac{1}{\sqrt{n}}\left(\begin{array}{llll}1 & 1 & \cdots & 1\end{array}\right)$ and $\left\{\left|\psi_{k}\right\rangle\right\}_{k>1}$ is an arbitrary orthonormal basis of the orthogonal subspace of $\left|\psi_{1}\right\rangle$. Since $A^{(i, j)}=A^{(j, i)}=B^{(i, j)}=B^{(j, i)}=0$ when $i \in\{1,2\}$ and $j \in\{0,3\}$, we have

$$
f(A, A+B)=f\left(A^{(0)}, A^{(0)}+B^{(0)}\right)+f\left(A^{(1)}, A^{(1)}+B^{(1)}\right)
$$

where

$$
(\cdot)^{(0)}=\left(\begin{array}{ll}
(\cdot)^{(0,0)} & (\cdot)^{(0,3)} \\
(\cdot)^{(3,0)} & (\cdot)^{(3,3)}
\end{array}\right), \quad(\cdot)^{(1)}=\left(\begin{array}{ll}
(\cdot)^{(1,1)} & (\cdot)^{(1,2)} \\
(\cdot)^{(2,1)} & (\cdot)^{(2,2)}
\end{array}\right) .
$$


We first calculate $f\left(A^{(0)}, A^{(0)}+B^{(0)}\right)$. We have

$$
\begin{aligned}
&\left(A^{(0)}\right)^{1 / 2}\left(A^{(0)}+B^{(0)}\right)\left(A^{(0)}\right)^{1 / 2}= \\
&\left(\begin{array}{c}
\frac{1}{4} \\
\frac{m^{2}}{4 n^{2}}
\end{array}\right)\left(\begin{array}{ll}
\frac{1}{4} & \frac{m^{2}}{4 n^{2}}
\end{array}\right) \otimes\left|\psi_{1}\right\rangle\left\langle\psi_{1}\left|+\left(\begin{array}{cc}
0 & 0 \\
0 & \left(\frac{n^{2}-m^{2}}{4 n^{2}(n-1)}\right)^{2}
\end{array}\right) \otimes \sum_{k=2}^{n}\right| \psi_{k}\right\rangle\left\langle\psi_{k}\right| .
\end{aligned}
$$

Then

$$
\begin{aligned}
f\left(A^{(0)}, A^{(0)}+B^{(0)}\right) & =\operatorname{Tr}\left(\left(\left(A^{(0)}\right)^{1 / 2}\left(A^{(0)}+B^{(0)}\right)\left(A^{(0)}\right)^{1 / 2}\right)^{1 / 2}\right) \\
& =\sqrt{\frac{1}{4^{2}}+\left(\frac{m^{2}}{4 n^{2}}\right)^{2}}+\frac{n^{2}-m^{2}}{4 n^{2}}=\frac{1}{2}-\frac{m^{2}}{4 n^{2}}+O\left(\frac{m^{4}}{n^{4}}\right) .
\end{aligned}
$$

In order to calculate $f\left(A^{(0)}, A^{(0)}+B^{(0)}\right)$, we first note that

$$
\left(A^{(1)}\right)^{1 / 2}\left(A^{(1)}+B^{(1)}\right)\left(A^{(1)}\right)^{1 / 2}=\left(\begin{array}{cc}
\left(A^{(1,1)}\right)^{2} & 0 \\
0 & \left(A^{(1,1)}\right)^{2}
\end{array}\right)+\left(\begin{array}{cc}
0 & i \frac{m}{4 n^{2}} A^{(1,1)} \\
-i \frac{m}{4 n^{2}} A^{(1,1)} & 0
\end{array}\right) .
$$

Then we use the Taylor expansion formula for square root of positive matrices: $\sqrt{\Lambda^{2}+Y}=$ $\Lambda+\chi[Y]-\chi\left(\chi[Y]^{2}\right)+O\left(Y^{3}\right)$ for any positive diagonal matrix $\Lambda$ and small $Y$ [75], where

$$
\chi[(\cdot)]_{i j}=\frac{(\cdot)_{i j}}{\Lambda_{i}+\Lambda_{j}} .
$$

Let $A^{(1)}=\Lambda$ such that $\Lambda_{1}=\frac{n^{2}+2 n-m^{2}}{8 n^{2}}$ and $\Lambda_{k}=\frac{n^{2}-2 n+m^{2}}{8 n^{2}(n-1)}$ for $k>1$, we find that

$$
f\left(A^{(1)}, A^{(1)}+B^{(1)}\right)=\frac{1}{2}-\left(\frac{m}{4 n^{2}}\right)^{2} \sum_{k=1}^{n} \frac{1}{4 \Lambda_{k}}+O\left(\frac{m^{3}}{n^{3}}\right)=\frac{1}{2}-\frac{m^{2}}{8 n^{2}}+O\left(\frac{m^{3}}{n^{3}}\right) .
$$

Therefore

$$
1-f(\mathcal{A}, \mathcal{A}+\delta \mathcal{A})^{2}=1-f(A, A+B)^{2}=\frac{3 m^{2}}{4 n^{2}}+O\left(\frac{m^{3}}{n^{3}}\right)
$$

which serves as an upper bound on the infidelity of thermodynamic codes under depolarizing noise due to Lemma 4.

\section{References}

[1] M. A. Nielsen and I. L. Chuang, Quantum Computation and Quantum Information (Cambridge University Press, 2010).

[2] D. Gottesman, in Quantum information science and its contributions to mathematics, Proceedings of Symposia in Applied Mathematics, Vol. 68 (2010) pp. 13-58.

[3] D. A. Lidar and T. A. Brun, Quantum error correction (Cambridge university press, 2013).

[4] B. Eastin and E. Knill, Restrictions on transversal encoded quantum gate sets, Physical Review Letters 102, 110502 (2009).

[5] S. Bravyi and R. König, Classification of topologically protected gates for local stabilizer codes, Physical Review Letters 110, 170503 (2013).

[6] F. Pastawski and B. Yoshida, Fault-tolerant logical gates in quantum error-correcting codes, Physical Review A 91, 012305 (2015).

[7] T. Jochym-O'Connor, A. Kubica, and T. J. Yoder, Disjointness of stabilizer codes and limitations on fault-tolerant logical gates, Physical Review X 8, 021047 (2018).

[8] D.-S. Wang, G. Zhu, C. Okay, and R. Laflamme, Quasi-exact quantum computation, Physical Review Research 2 (2020).

[9] P. Hayden, S. Nezami, S. Popescu, and G. Salton, Error correction of quantum reference frame information, PRX Quantum 2 (2021).

[10] P. Faist, S. Nezami, V. V. Albert, G. Salton, F. Pastawski, P. Hayden, and J. Preskill, Continuous symmetries and approximate quantum error correction, Physical Review X 10 (2020). 
[11] J. Preskill, Quantum clock synchronization and quantum error correction, (2000), arXiv:quant-ph/0010098 [quant-ph] .

[12] M. P. Woods and Á. M. Alhambra, Continuous groups of transversal gates for quantum error correcting codes from finite clock reference frames, Quantum 4, 245 (2020).

[13] A. Almheiri, X. Dong, and D. Harlow, Bulk locality and quantum error correction in ads/cft, Journal of High Energy Physics 2015, 163 (2015).

[14] F. Pastawski, B. Yoshida, D. Harlow, and J. Preskill, Holographic quantum error-correcting codes: Toy models for the bulk/boundary correspondence, Journal of High Energy Physics 2015, 149 (2015).

[15] D. Harlow and H. Ooguri, Constraints on symmetries from holography, Physical Review Letters 122, 191601 (2019).

[16] D. Harlow and H. Ooguri, Symmetries in quantum field theory and quantum gravity, (2018), arXiv:1810.05338 [hep-th] .

[17] T. Kohler and T. Cubitt, Toy models of holographic duality between local hamiltonians, Journal of High Energy Physics 2019, 17 (2019).

[18] M. Gschwendtner, R. König, B. Şahinoğlu, and E. Tang, Quantum error-detection at low energies, Journal of High Energy Physics 2019, 21 (2019).

[19] F. G. S. L. Brandão, E. Crosson, M. B. Şahinoğlu, and J. Bowen, Quantum error correcting codes in eigenstates of translation-invariant spin chains, Physical Review Letters 123, 110502 (2019).

[20] C. Bény and O. Oreshkov, General conditions for approximate quantum error correction and near-optimal recovery channels, Physical Review Letters 104, 120501 (2010).

[21] P. Hayden, M. Horodecki, A. Winter, and J. Yard, A decoupling approach to the quantum capacity, Open Systems \& Information Dynamics 15, 7 (2008).

[22] C. Bény, Z. Zimborás, and F. Pastawski, Approximate recovery with locality and symmetry constraints, (2018), arXiv:1806.10324 [quant-ph] .

[23] V. Giovannetti, S. Lloyd, and L. Maccone, Advances in quantum metrology, Nature Photonics 5, 222 (2011).

[24] C. L. Degen, F. Reinhard, and P. Cappellaro, Quantum sensing, Reviews of Modern Physics 89, 035002 (2017).

[25] D. Braun, G. Adesso, F. Benatti, R. Floreanini, U. Marzolino, M. W. Mitchell, and S. Pirandola, Quantum-enhanced measurements without entanglement, Reviews of Modern Physics 90, 035006 (2018).

[26] L. Pezzè, A. Smerzi, M. K. Oberthaler, R. Schmied, and P. Treutlein, Quantum metrology with nonclassical states of atomic ensembles, Reviews of Modern Physics 90, 035005 (2018).

[27] S. Pirandola, B. R. Bardhan, T. Gehring, C. Weedbrook, and S. Lloyd, Advances in photonic quantum sensing, Nature Photonics 12, 724 (2018).

[28] B. Escher, R. de Matos Filho, and L. Davidovich, General framework for estimating the ultimate precision limit in noisy quantum-enhanced metrology, Nature Physics 7, 406 (2011).

[29] R. Demkowicz-Dobrzański, J. Kołodyński, and M. Guţă, The elusive heisenberg limit in quantum-enhanced metrology, Nature Communications 3, 1063 (2012).

[30] R. Demkowicz-Dobrzański and L. Maccone, Using entanglement against noise in quantum metrology, Physical Review Letters 113, 250801 (2014).

[31] H. Yuan and C.-H. F. Fung, Quantum parameter estimation with general dynamics, npj Quantum Information 3, 1 (2017).

[32] R. Demkowicz-Dobrzański, J. Czajkowski, and P. Sekatski, Adaptive quantum metrology under general markovian noise, Physical Review X 7, 041009 (2017).

[33] S. Zhou, M. Zhang, J. Preskill, and L. Jiang, Achieving the heisenberg limit in quantum metrology using quantum error correction, Nature Communications 9, 78 (2018).

[34] S. Zhou and L. Jiang, Asymptotic theory of quantum channel estimation, PRX Quantum 2 (2021).

[35] A. Fujiwara and H. Imai, A fibre bundle over manifolds of quantum channels and its application to quantum statistics, Journal of Physics A: Mathematical and Theoretical 41, 255304 (2008).

[36] M. Hayashi, Comparison between the cramer-rao and the mini-max approaches in quantum channel estimation, Communications in Mathematical Physics 304, 689 (2011). 
[37] H. Yuan and C.-H. F. Fung, Fidelity and fisher information on quantum channels, New Journal of Physics 19, 113039 (2017).

[38] V. Katariya and M. M. Wilde, Geometric distinguishability measures limit quantum channel estimation and discrimination, Quantum Information Processing 20 (2021).

[39] G. Gour and R. W. Spekkens, The resource theory of quantum reference frames: manipulations and monotones, New Journal of Physics 10, 033023 (2008).

[40] I. Marvian and R. W. Spekkens, How to quantify coherence: Distinguishing speakable and unspeakable notions, Physical Review A 94, 052324 (2016).

[41] I. Marvian and R. W. Spekkens, Extending noether's theorem by quantifying the asymmetry of quantum states, Nature communications 5, 1 (2014).

[42] K. Fang and Z.-W. Liu, No-go theorems for quantum resource purification, Physical Review Letters 125, 060405 (2020).

[43] B. Regula, K. Bu, R. Takagi, and Z.-W. Liu, Benchmarking one-shot distillation in general quantum resource theories, Physical Review A 101 (2020).

[44] I. Marvian, Coherence distillation machines are impossible in quantum thermodynamics, Nature Communications 11, 1 (2020).

[45] B. Schumacher, Sending entanglement through noisy quantum channels, Physical Review A 54, 2614 (1996).

[46] A. Gilchrist, N. K. Langford, and M. A. Nielsen, Distance measures to compare real and ideal quantum processes, Physical Review A 71, 062310 (2005).

[47] A. Kubica and R. Demkowicz-Dobrzański, Using quantum metrological bounds in quantum error correction: A simple proof of the approximate eastin-knill theorem, Physical Review Letters 126 (2021).

[48] E. M. Kessler, I. Lovchinsky, A. O. Sushkov, and M. D. Lukin, Quantum error correction for metrology, Physical Review Letters 112, 150802 (2014).

[49] G. Arrad, Y. Vinkler, D. Aharonov, and A. Retzker, Increasing sensing resolution with error correction, Physical Review Letters 112, 150801 (2014).

[50] W. Dür, M. Skotiniotis, F. Fröwis, and B. Kraus, Improved quantum metrology using quantum error correction, Physical Review Letters 112, 080801 (2014).

[51] X.-M. Lu, S. Yu, and C. Oh, Robust quantum metrological schemes based on protection of quantum fisher information, Nature Communications 6, 7282 (2015).

[52] F. Reiter, A. S. Sørensen, P. Zoller, and C. Muschik, Dissipative quantum error correction and application to quantum sensing with trapped ions, Nature Communications 8, 1822 (2017).

[53] P. Sekatski, M. Skotiniotis, J. Kołodyński, and W. Dür, Quantum metrology with full and fast quantum control, Quantum 1, 27 (2017).

[54] T. Kapourniotis and A. Datta, Fault-tolerant quantum metrology, Physical Review A 100, 022335 (2019).

[55] D. Layden and P. Cappellaro, Spatial noise filtering through error correction for quantum sensing, npj Quantum Information 4, 30 (2018).

[56] D. Layden, S. Zhou, P. Cappellaro, and L. Jiang, Ancilla-free quantum error correction codes for quantum metrology, Physical Review Letters 122, 040502 (2019).

[57] S. Zhou and L. Jiang, Optimal approximate quantum error correction for quantum metrology, Physical Review Research 2, 013235 (2020).

[58] C. W. Helstrom, Quantum detection and estimation theory (Academic press, 1976).

[59] A. S. Holevo, Probabilistic and statistical aspects of quantum theory, Vol. 1 (Springer Science \& Business Media, 2011).

[60] M. G. Paris, Quantum estimation for quantum technology, International Journal of Quantum Information 7, 125 (2009).

[61] S. L. Braunstein and C. M. Caves, Statistical distance and the geometry of quantum states, Physical Review Letters 72, 3439 (1994).

[62] H. Yuen and M. Lax, Multiple-parameter quantum estimation and measurement of nonselfadjoint observables, IEEE Transactions on Information Theory 19, 740 (1973).

[63] V. Giovannetti, S. Lloyd, and L. Maccone, Quantum metrology, Physical Review Letters 96, 010401 (2006).

[64] J. Kołodyński and R. Demkowicz-Dobrzański, Efficient tools for quantum metrology with uncorrelated noise, New Journal of Physics 15, 073043 (2013). 
[65] A. Streltsov, G. Adesso, and M. B. Plenio, Colloquium: Quantum coherence as a resource, Reviews of Modern Physics 89, 041003 (2017).

[66] K. Fang and Z.-W. Liu, No-go theorems for quantum resource purification: new approach and channel theory, (2021), arXiv:2010.11822 [quant-ph] .

[67] B. Regula and R. Takagi, One-shot manipulation of dynamical quantum resources, (2020), arXiv:2012.02215 [quant-ph] .

[68] Y. Ouyang, N. Shettell, and D. Markham, Robust quantum metrology with explicit symmetric states, (2019), arXiv:1908.02378 [quant-ph].

[69] D. Gottesman, Quantum fault tolerance in small experiments, (2016), arXiv:1610.03507 [quant-ph] .

[70] Z.-W. Liu and A. Winter, Resource theories of quantum channels and the universal role of resource erasure, (2019), arXiv:1904.04201 [quant-ph] .

[71] Y. Liu and X. Yuan, Operational resource theory of quantum channels, Physical Review Research 2, 012035 (2020)

[72] Y. Yang, Y. Mo, J. M. Renes, G. Chiribella, and M. P. Woods, Covariant quantum error correcting codes via reference frames, (2020), arXiv:2007.09154 [quant-ph] .

[73] H. Komiya, Elementary proof for sion's minimax theorem, Kodai Mathematical Journal 11, 5 (1988).

[74] M. do Rosário Grossinho and S. A. Tersian, An introduction to minimax theorems and their applications to differential equations, Vol. 52 (Springer Science \& Business Media, 2001).

[75] P. Del Moral and A. Niclas, A taylor expansion of the square root matrix function, Journal of Mathematical Analysis and Applications 465, 259 (2018). 